\title{
Josefina San Martín Bacaicoa
}

\author{
Francisco MARAVER EYZAGUIRRE ${ }^{(1-2)}$
}

(1) Escuela Profesional de Hidrología Médica e Hidroterapia. Facultad de Medicina. Universidad Complutense de Madrid. Plaza Ramón y Cajal s/n, 28040 Madrid (España).

${ }^{(2)}$ Departamento Medicina Física y Rehabilitación. Hidrología Médica.

Facultad de Medicina. Universidad Complutense de Madrid. Plaza Ramón y Cajal s/n, 28040 Madrid (España).

fmaraver@med.ucm.es

\begin{abstract}
Resumen
Josefina San Martín Bacaicoa, tras concurso oposición ordinario, celebrado a finales de 1986, se convierte en el cuarto catedrático de Hidrología Médica en la historia de la Universidad española. Desde que alcanzara el Grado de Medicina y Cirugía hasta ese momento, su actividad profesional asistencial, docente e investigadora se ha desarrollado en el ámbito de dos especialidades médica: la Rehabilitación, en el Centro Nacional de Rehabilitación; y la Hidrología Médica, en la Cátedra de Hidrología Médica de la Facultad de Medicina de la Universidad Complutense de Madrid y en diferentes Balnearios, Panticosa, Arnedillo, San Juan de Campos y Molgas. Ya catedrática, se dedica en exclusividad a la Hidrología desarollando una importante labor asociativa, organizativa y de internacionalización de la especialidad. Así mismo, mantiene una constante actividad académica en las Reales Academias Nacionales de Medicina y Farmacia donde ingresa como académica correspondiente en los años 1989 y 1996, respectivamente
\end{abstract}

Palabras clave: Josefina San Martín, Hidrología Médica, Rehabilitación, Especialidades Médicas, Cátedras de Universidad, Sociedades Científicas, Revistas Médicas

\section{Josefina San Martín Bacaicoa}

\begin{abstract}
Josefina San Martín Bacaicoa, after an ordinary competitive examination in 1986, became the fourth professor of Medical Hydrology in the history of the Spanish Universities. The period between her bachiller degree in Medicine and Surgery and that moment is dedicated to her professional medical practice, teaching and investigation which has always been related to her two medical specializations: Rehabilitation Therapy in Centro Nacional de Rehabilitación and Medical Hydrology within the Chair in Medical Hydrology of the
\end{abstract}


Faculty of Medicine in the Complutense University of Madrid and in several spa establishments such as Panticosa, Arnedillo, San Juan de Campos and Molgas. Since she achieved the chair, she is solely dedicated to Hydrology and carries out an important role in associations, organization and internationalization related to this speciality. At the same time, she has constant academic activity in the Royal National Academies of both Medicine and Pharmacy where she is corresponding member since the years 1989 and 1996 respectively.

Key words: Josefina San Martín, Medical Hydrology, Rehabilitation, Medical Specialization, University Chairs, Scientific Society, Medical Journals

REFERENCIA NORMALIZADA

Maraver F. Josefina San Martín Bacaicoa. Balnea, 2012, 7, 133-182.

\section{INTRODUCCIÓN}

Independientemente de los sentimientos de consideración, respeto y gratitud que la figura de Josefina San Martín me evoca, abordar esta breve semblanza ha supuesto para mí un verdadero reto personal ya que se escapa a mis normales quehaceres profesionales.

No obstante, el hecho de ser un espectador privilegiado de parte de su vida académica tanto como alumno de la Escuela Profesional de Hidrología Médica, directora de mi Tesis doctoral y como compañera en la Facultad de Medicina de la UCM, pero sobre todo, gracias a sus aclaraciones y generosa colaboración, he podido afrontarla.

El trabajo se desarrolla en cuatro partes: una, dedicada a sus primeros años, es decir, desde su nacimiento en Navarra hasta su traslado a Madrid para iniciar los estudios de la licenciatura de Medicina; la segunda, corresponde a la etapa como estudiante de Medicina, desde sus comienzos hasta alcanzar el grado de doctor; la tercera, dedicada a su faceta como médico rehabilitadora y la cuarta, a su consagración a la Hidrología Médica, tanto en actividades docentes, investigadoras, asistenciales y asociativas, como académicas en las Reales Academias Nacionales de Medicina y Farmacia.

A estas, he añadido, además de la bibliografía, un anexo en el que se recoge la producción científica más relevante de la doctora San Martín, es decir: las publicaciones en revistas científicas nacionales e internacionales, libros, capítulos de libros, aportaciones a congresos nacionales e internacionales y las Tesis Doctorales y Tesinas dirigidas. 


\section{PRIMEROS AÑOS}

Nació en Ulzurrun, Valle de Ollo (Navarra) el 22 de julio de 1934 en la casa familiar junto a la iglesia de San Martín. El concejo de Ulzurrun es uno de los ocho que forman el municipio de Ollo situado en el diapiro del mismo nombre. Desde el punto de vista geográfico se haya equidistante de la capital Pamplona y de los Parques Naturales de Aralar y Urbasa-Andía ${ }^{48}$.

Según sus informaciones, en las proximidades, en dirección noroeste se encuentra la Sierra de Aralar con su conocido Santuario de San Miguel de Aralar, donde Navarro-Villoslada, sitúa el origen de los vascos, ella siempre ha considerado el lugar como algo entrañable con el que se siente totalmente identificada y al que acude siempre que puede.

Miembro de una familia numerosa, fue la novena de diez hermanos, quedando huérfana de padre a la edad de nueve años. En esta situación, su hernana Isabel, la primogénita, traslada el domicilio familiar a Pamplona, donde Josefina hará sus estudios de primaria y cursará el bachillerato en el Instituto Femenino "Príncipe de Viana" de Pamplona entre los años 1945-1951 (figura 1).

Figura 1 - Instituto Femenino "Príncipe de Viana" de Pamplona. Promoción $1951^{8}$

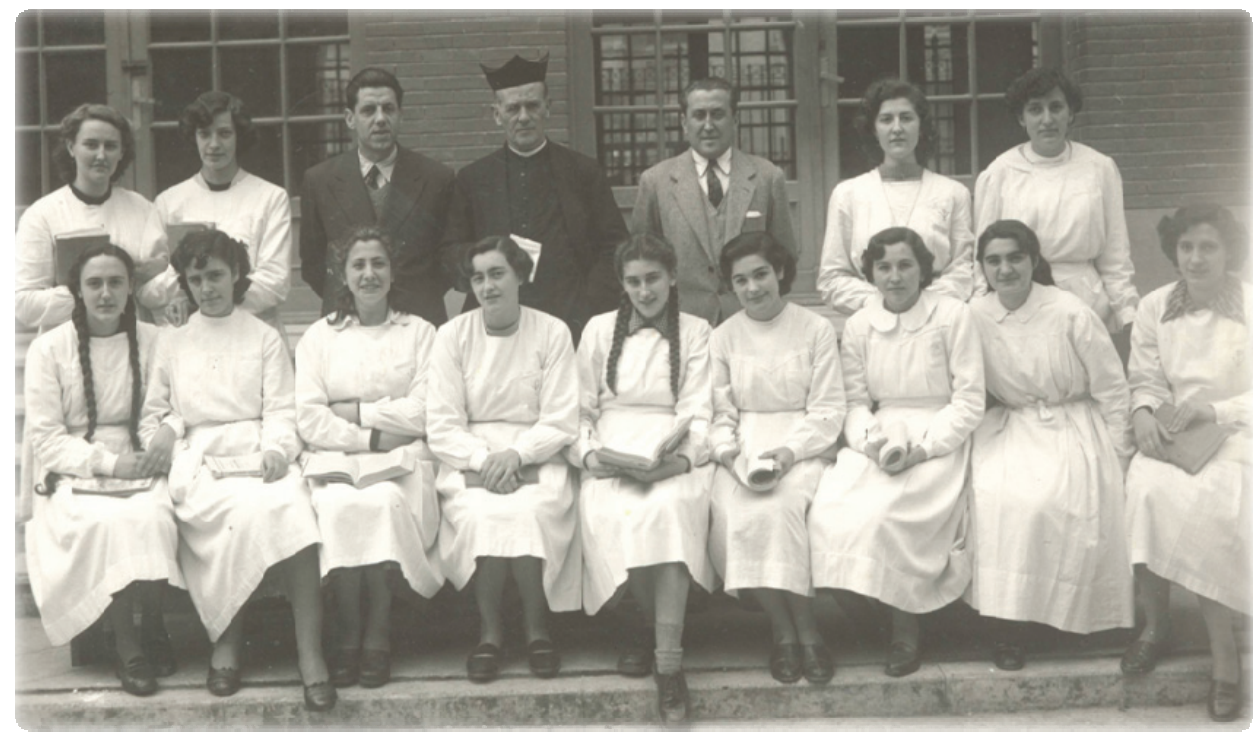

Con 18 años, en septiembre 1952, aprueba el examen de Estado para la obtención del Título de Bachiller Superior, imprescindible entonces para acceder a la Universidad. Traslada su residencia a Zaragoza donde inicia en su Universidad, los estudios de la Licenciatura de Ciencias Químicas en el curso académico 1952-53. 
No obstante, problemas de salud, de tipo respiratorio, le impedirán continuar en el curso siguiente. Siempre recordará, la expectación que supuso, entre sus compañeras del Colegio Mayor Santa Isabel, el traslado en ambulancia desde Zaragoza al Valle de Ollo para su total restablecimiento durante gran parte del año 1954.

Superada esta etapa decide hacer la carrera de Magisterio, matriculándose como alumna libre en la Escuela de Magisterio de Pamplona donde hace los dos primeros años, pero habiéndose trasladado el domicilio familiar a San Sebastian, cursa el tercer año, ya como alumna oficial, en la Escuela de Magisterio de Guipúzcoa de dicha capital, obteniendo el Título de Maestra de Primera Enseñanza, carrera que nunca ejercerá (figura 2).

Figura 2 - Retrato juvenil de Josefina San Martín Bacaicoa ${ }^{8}$

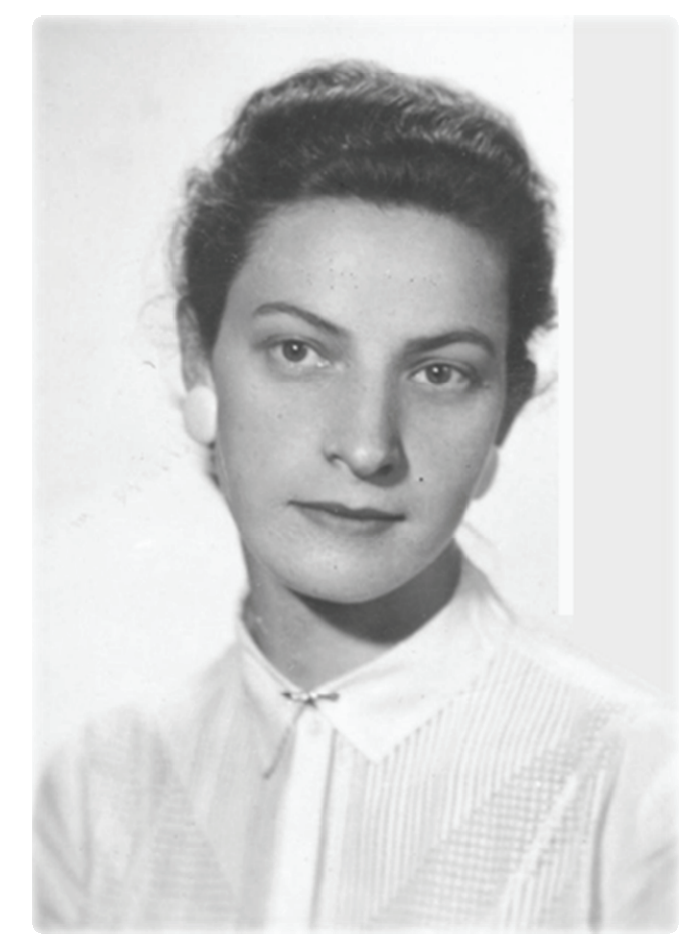

Iniciada la década de los sesenta decide realizar estudios de perfeccionamiento del idioma Inglés, instalándose en Londres durante los años 1961, 1962 y 1963, donde asiste a las County Council School of Languages de Londres y a la Golders Green School of Languages obteniendo, por examen, los siguientes títulos: "Cambridge Certificate in English" y "Certificate in English for foreigners of the Society of Arts and Commerce". 
Según sus propias palabras, tras este período, decide trasladarse a Madrid con el firme propósito de realizar la carrera de Medicina para dedicarse a la Rehabilitación de niños discapacitados. Ya aquí, y para ayudarse económicamente, colabora con una academia de idiomas impartiendo clases de inglés, de esta manera conocerá como alumno al que será su futuro tutor y mentor en la Universidad Complutense, el nuevo Catedrático de Hidrología Médica Manuel Armijo Valenzuela.

\section{JOSEFINA SAN MARTÍN BACAICOA ESTUDIANTE DE MEDICINA}

Comenzó sus estudios de Medicina en el curso académico 1964-65. Desde su incorporación como alumna, a la Facultad de Medicina de la Universidad Complutense, Josefina San Martín estuvo vinculada al laboratorio de la Cátedra de Hidrología Médica entonces en los locales de Farmacología.

En estos años el equipo de la Cátedra, además del Prof. Armijo Valenzuela, estaba compuesto por don José San Román Terán, Alfonso Velasco Martín, José Miguel Arévalo Alonso y Francisco Armijo Castro. En enero de 1965 Luís Pablo Rodríguez Rodríguez se incorpora procedente de la Facultad de Medicina de Valladolid como Prof Adjunto Interino y gana la plaza por oposición en mayo del año

Figura 3 - San Martín, Armijo y Rodríguez. Vichy-Francia, verano de $1966^{8}$

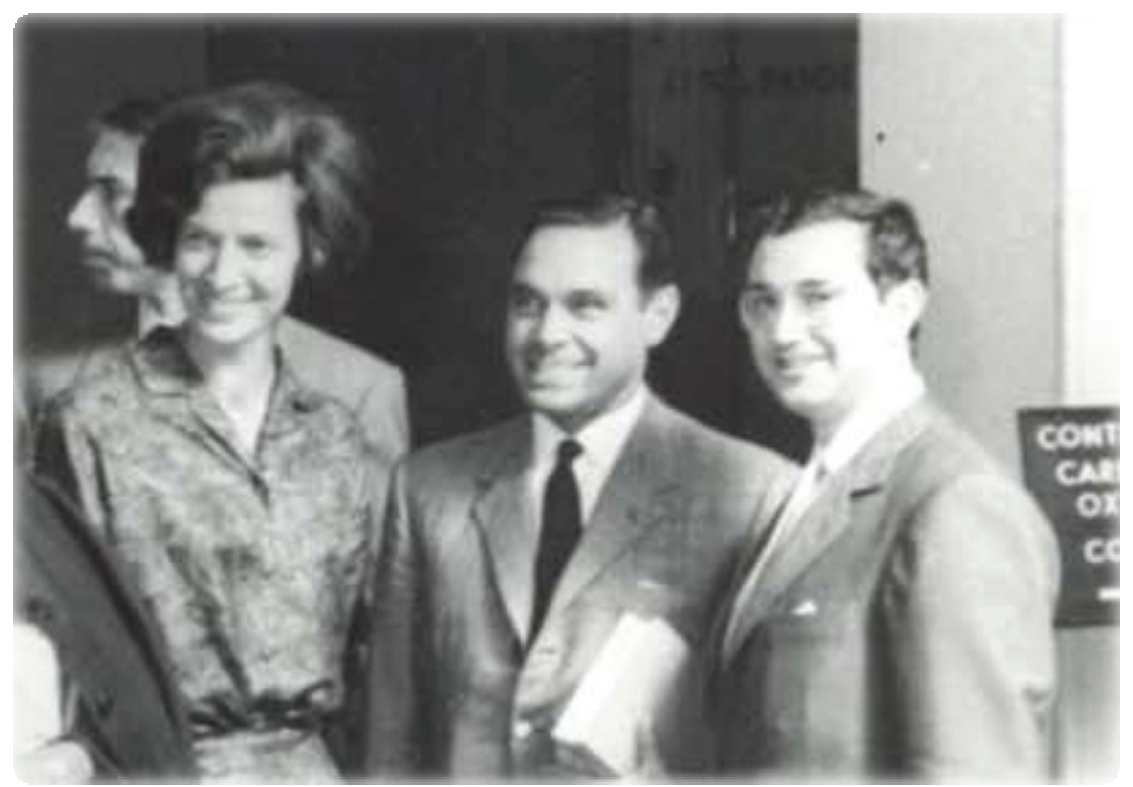


siguiente. Así mismo, hay que señalar la creación de la Escuela Profesional de Hidrología Médica e Hidroterapia, aprobada en 1968, aunque puesta en funcionamiento diez años más tarde $\mathrm{e}^{3-4-29.32-33-37-38-45}$.

Durante toda la carrera disfrutaró de numerosas estancias en el extranjero, por tanto, resultó una verdadera precursora de la filosofía del Programa Erasmus, con el consiguiente enriquecimiento intelectual recibido en ellas. Cabe destacar ${ }^{22-48}$ :

- Curso Internacional de Verano - Institut D'Hydrologie et de Climatologie. Université de Clermont-Ferrand (Francia), bajo la tutela del Prof. Cuvelier.VII-1965.

- Curso Internacional de Verano - Institut D'Hydrologie et de Climatologie. Université de Clermont- Ferrand (Francia), bajo la tutela del Prof. Cuvelier. IX-1966 (figura 3).

- Hospital Santa María Adelaida de Torino (Italia), Servicios de Rehabilitación y Pediatría. Verano 1969.

- Bispebjerg Hospital de Copenhagen (Dinamarca). Departamento de Medicina Interna. Agosto 1970.

- Estancia en el Hospital de Popo-Cabaca (Zaire) diciembre 1970 (figura 4).

Incluso siendo ya Licenciada en Medicina y Cirugía, realizó otros dos en:

- Saint Mary of Nazareth Hospital de Chicago (USA), bajo la tutela del Prof. Bicari. 7-VII-71 a 6-IX-71.

- Rheumatic Deseases Hospital de Bath (Inglaterra), bajo la tutela del Prof. Yeomans. Verano 1974.

Finalizada la carrera, el tres de noviembre de 1971, supera el examen de Grado con la calificación de sobresaliente y obtiene el nombramiento de Médico Interno de Hidrología Médica del Departamento de Radiología y Fisioterapia del que en esos años es director el Profesor Manuel Armijo Valenzuela.

Esto le permite, por un lado, afrontar las asignaturas del Doctorado en la Facultad de Medicina de la UCM, concretamente las de Historia de la Medicina, Anestesia y Reanimación, Hidrología Médica y Reumatología en las que consigue tres matrículas de Honor, y por otro aproximarse al Servicio de Radiología del Hospital Clínico San Carlos que dirigía el Profesor Miguel Gil Gayarre, al que considerará siempre como uno de sus Maestros, guardando hondo recuerdo de esta época en la se formó junto a un equipo de jóvenes promesas de la Universidad española en el área de conocimiento de Radiología y Medicina Física (María Teresa Delgado, Carmen Galván, Manuel Martínez Murillo, Claudio Otón...).

Desde el Curso 1972-73, simultaneará su trabajo en el Centro Nacional de Rehabilitación por las mañanas con el de la Cátedra de Hidrología Médica, por las tardes, hasta que el 15 de julio de 1975 alcanza el grado de doctor con la calificación de Sobresaliente cum laude, con la lectura y defensa de la memoria académica titulada "Relación entre la Conductividad Específica y la Densidad relativa de las Aguas Minero-Medicinales y su Residuo Seco", dirigida por el Prof. Armijo Valenzuela. 
Figura 4 - Hospital de Popo-Cabaca - Zaire. Diciembre $1970^{8}$

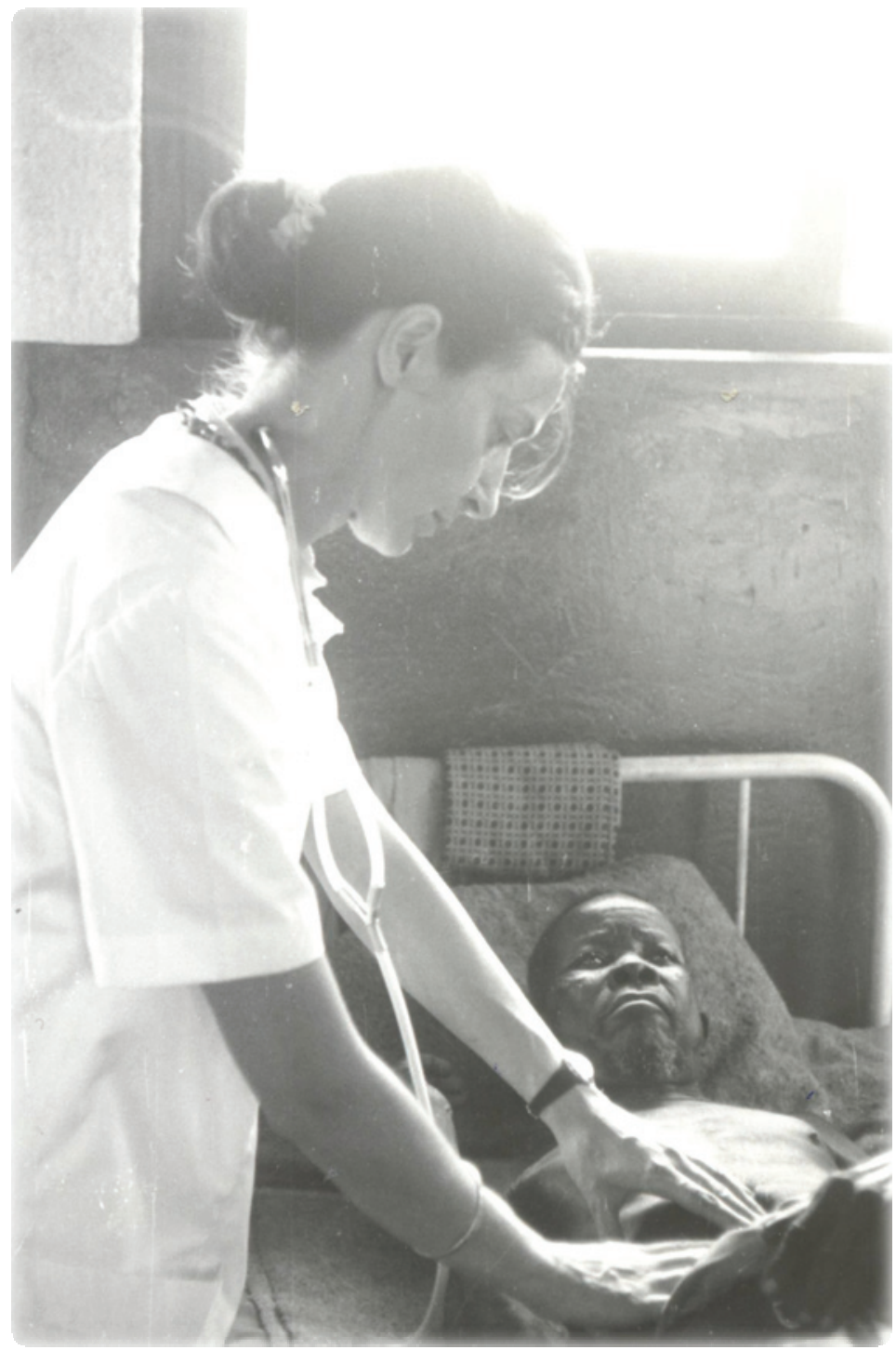

Con posterioridad cursará en la Facultad de Farmacia, durante el Curso 1975-76, la asignatura del doctorado de Análisis Químico al objeto de cumplir los requisitos para presentarse a una posible convocatoria de oposiciones de médicos del cuerpo de baños. 
Como culminación de este apartado se incluye la figura 5, que muestra la solemne ceremonia de presentación de doctores en el acto de apertura de curso académico 1976-77, primera presidida por los nuevos Reyes de España, en el marco incomparable del gran paraninfo de San Bernardo, inaugurado en 1852 siendo reina su tatarabuela Isabel $\mathrm{II}^{16}$, observandose el rico protocolo complutense $\mathrm{e}^{17}$.

Figura 5 - Solemne apertura del Curso 1976-77 ${ }^{8}$.

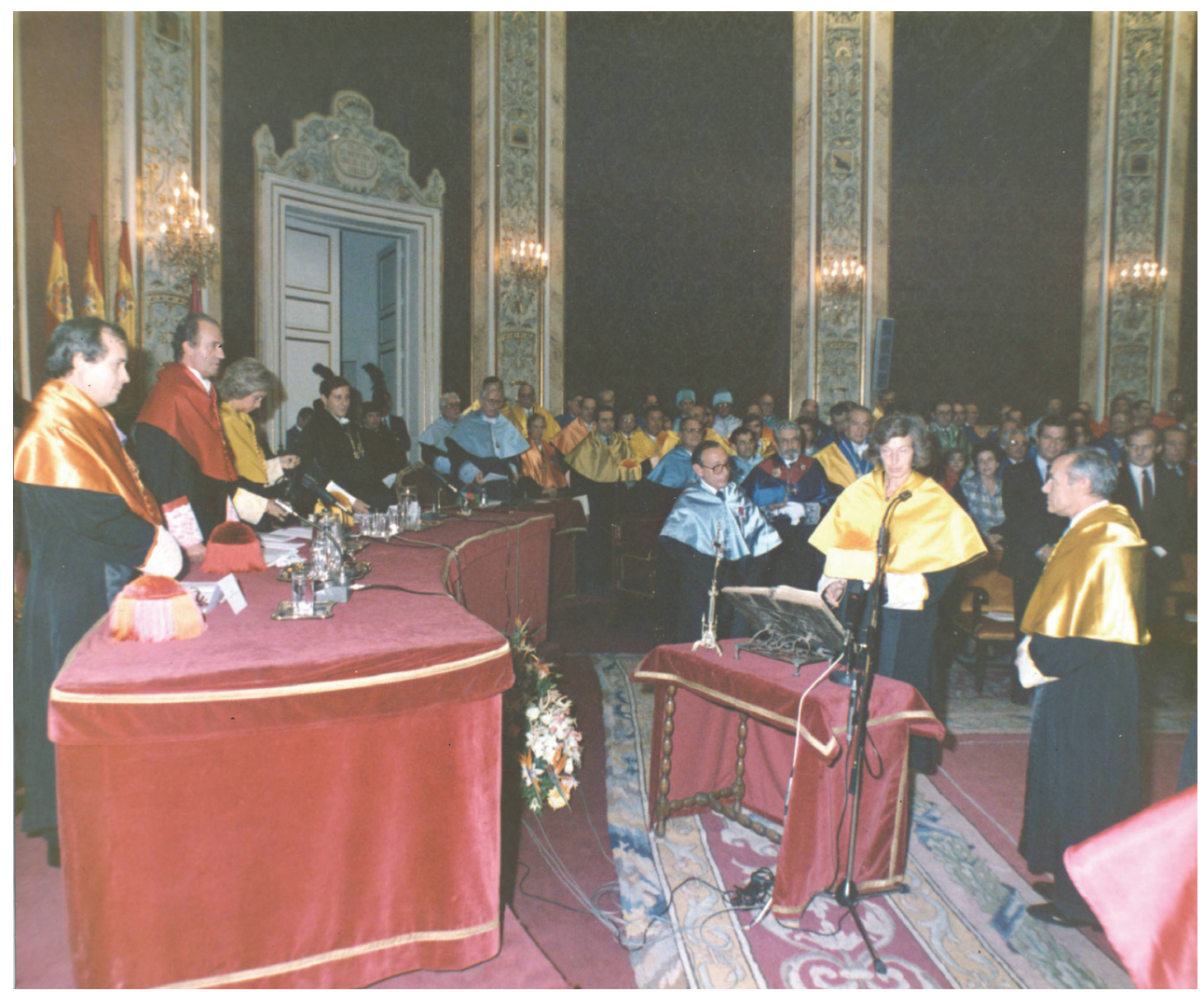

\section{JOSEFINA SAN MARTÍN BACAICOA MÉDICO REHABILITADORA}

Como acabamos de señalar, previo concurso, a partir de curso 1972-73 accede a la plaza de médico residente en el Centro Nacional de Rehabilitación; verdadero centro de referencia en nuestro país de esta especialidad bajo la dirección del Dr. Antonio Roldán Álvarez. En esta época prestaban servicio doce médicos rehabilitadores, dos reumatólogos, un neurólogo, un médico internista y cuatro traumatólogos. 
En 1974 consigue el título de médico especialista en rehabilitación, desempeñando la plaza de médico adjunto desde 1974, pasando a ser funcionario de carrera en noviembre de 1978. En 1981 promociona a Jefe de sección hasta su excedencia voluntaria en 1984 para dedicarse en exclusividad a la Hidrología Médica.

En este período realiza numerosos cursos de formación destacando el de la Escuela Profesional de Reumatología, durante los cursos 1976-77 y 1977-78, obteniendo el correspondiente título de Médico especialista en 1979.

También y por Concurso Oposición Libre de Méritos, obtiene una plaza de Médico de zona de la Seguridad Social en el Ambulatorio Hermanos Aznar de Madrid, del que solicitó baja por excedencia voluntaria.

Como docente fue profesora entre otras instituciones de: la Escuela de Médicos Especialistas en Rehabilitación del Centro Nacional de Rehabilitación de la Dirección General de Sanidad y de la Universidad Complutense de Madrid, desde 1974 a 1981; la Escuela de Fisioterapia del Centro Nacional de Rehabilitación, desde 1974 a 1984; la Escuela de A.T.S. de San Francisco de Asís de Madrid, desde 1972 a 1980 y Médico encargado de la Unidad de Investigación y Docencia y Coordinadora de la Unidad Docente del Centro Nacional de Rehabilitación de la Dirección General de Sanidad, desde 1979 a 1984.

Por otra parte como miembro de la Sociedad Española de Rehabilitación, desempeñó el cargo de Secretaria General durante los años 1978-81, siendo Presidente el Dr. Antonio Roldan Álvarez, perteneciendo al Consejo de Redacción de la revista Rehabilitación órgano de expresión de la citada Sociedad.

Desde 1980 a 1985, actuó como representante del Ministerio de Universidades e Investigación, en la Comisión Nacional de Rehabilitación y en 1991 ejerció la Presidencia del Comité Científico del VI Internacional Rehabilitation Medicine Association Congress \& XIV Congreso Nacional de la Sociedad Española de Rehabilitación IRMA VI - MADRID $90^{48}$, en el transcurso del cual se celebró un simposio dedicado a la Hidrología Médica en Rehabilitación ${ }^{51}$.

\section{JOSEFINA SAN MARTÍN BACAICOA MÉDICO HIDRÓLOGA}

\section{Docencia}

Como docente en Hidrología Médica, el "cursus honorum" de Josefina San Martín e impecable ${ }^{48}$ :

Médico Interno de Hidrología Médica, desde octubre de 1971 a septiembre de 1974.

Prof. Colaborador Contratado adscrito a la Cátedra de Hidrología Médica, hasta 1973. 
Prof. Ayudante de Clases prácticas adscrito a la Cátedra de Hidrología Médica, desde octubre de 1973 a septiembre de 1975.

Prof. Adjunto Interino de Hidrología Médica, desde octubre de 1975 a septiembre de 1978.

Prof. Adjunto Numerario de Hidrología Médica, desde septiembre de 1978 a septiembre de 1983.

Prof. Titular de Universidad de Hidrología Médica, desde septiembre de 1983 a enero 1987.

Catedrático de Universidad de Radiología y Medicina Física (Hidrología Médica), desde enero 1987 a septiembre 2004.

Prof. Emérito, desde octubre de 2005 a septiembre 2008.

Actividad Docente:

En la UCM:

\section{Estudios de Licenciatura}

- Medicina

Asignatura Optativa Hidrología y Climatología Médica: responsable desde Curso $1994-95$ a 19992000 y 2001-02 a 2003-2004.

- Ciencia y Tecnología de los Alimentos

Asignatura Optativa Aguas Minerales y Mineromedicinales de consumo: responsable desde Curso 1994-95 a 19992000.

\section{Doctorado}

Asignatura del Doctorado Hidrología Médica: participa desde Curso 1971-72 a 1988-89

Curso Monográfico del Doctorado Hidroterapia: responsable desde Curso 198485 a1988-89

Programa de Doctorado "Hidrología Médica" (RD 537/1988 y 778/1998 - 32 Créditos): participa desde Curso 1989-90 a 1993-94 y Coordinadora principal del programa desde el Curso 1994-95 a 19992000 y 2001-02 a 2003-2004.

\section{Escuela Profesional de Hidrología Médica e Hidroterapia}

Cursos de formación de médicos especialistas en Hidrología Médica $1^{\circ}$ y $2^{\circ}$ Curso: participa desde Curso 1978-79 a 1993-94 y responsable desde Curso 1994-95 a 1999-2000 (figura 6).

\section{Títulos Propios}

Técnicas Balneoterápicas: Curso 1994-95

Terapia por el Mar y el Clima Marino: Curso 1996-97

Aguas Envasadas: Curso 1998-99 


\section{Universidad para los Mayores}

Programa de Ciencias de la Salud - Educación para la Salud: Hábitos saludables en la edad adulta: participa desde Curso 2000-01 hasta la actualidad

Figura 6 - Termas Pallarés-Alhama de Aragón, primavera de 1988

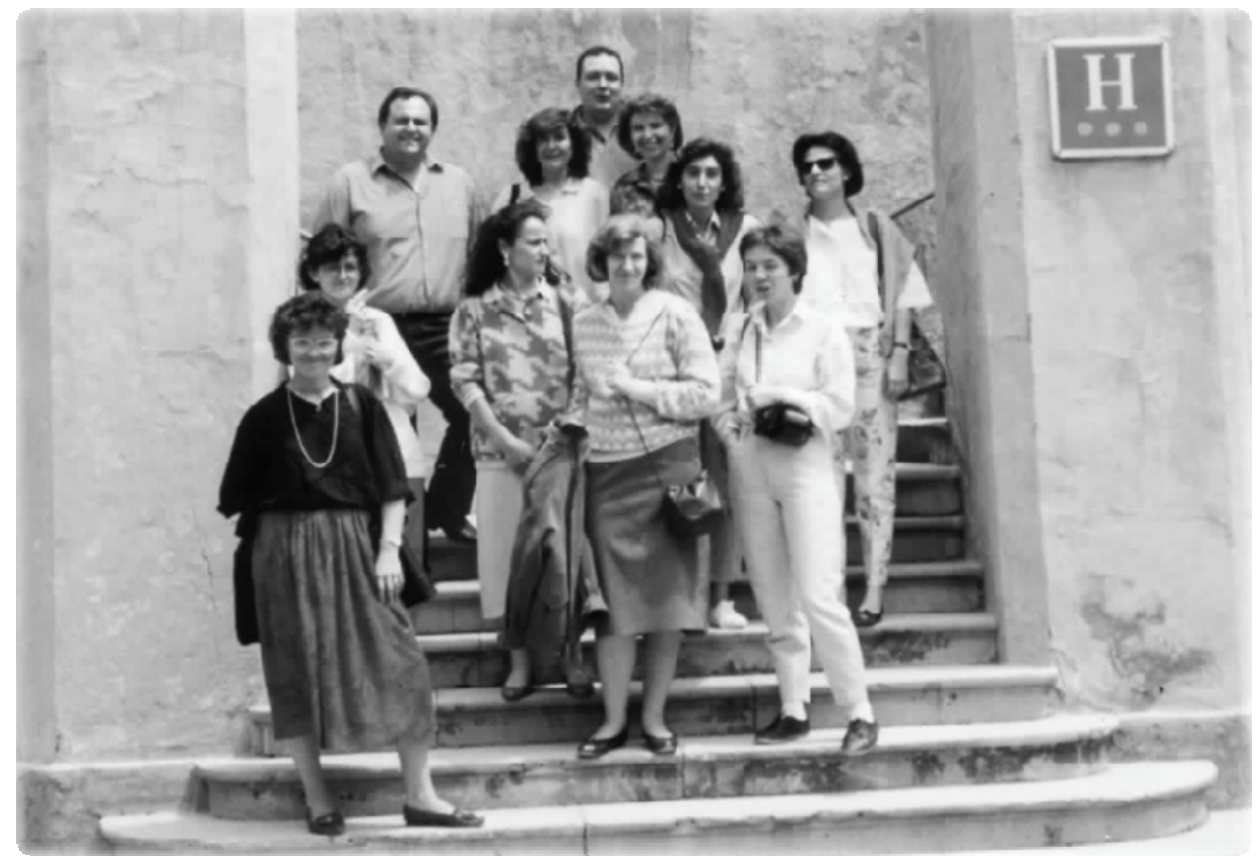

Cargos Académicos:

Directora de la Escuela Profesional de Hidrología Médica e Hidroterapia 4-XI1994 a 30-IX-2000 (ya que a requerimiento propio se le concede un año sabático Curso 2000-01).

\section{En otras Universidades:}

Universidad de Nacional de Santiago del Estero y Universidad Nacional del Comahue. Primer Curso y Simposio de Reumatología e Hidrología Médica. Santiago del Estero 1980.

Universidad de Murcia. IV Curso Internacional de estudio y tratamiento del dolor. Murcia 1988.

U.N.E.D. - Universidad de Verano. I Jornadas sobre el futuro del Termalismo. Calatayud 1989.

Universidad de Salamanca. Curso sobre Agua y Salud Pública. Salamanca 1991. 
Universidad de Málaga. Curso Seminario de Talasoterapia, Climatoterapia e Hidrología Médica. Málaga 1992.

Universidad de Tras-os-Montes e Alto Douro. Cursos de Verano. ChavesPortugal 1993 y 1994.

Universidad de Zaragoza. Postgrado de Medicina Naturista. Zaragoza 1993 y1994.

Universidad de La Coruña. Symposium Internacional de Investigación en Hidrología Médica. Balneario de Mondariz 1995.

Universidad de Sevilla. IV Curso de puesta al día en el diagnóstico y tratamiento del dolor de espalda. Sevilla 1997.

Universidad de La Coruña. Cursos de Doctorado - Significado del Termalismo en Galicia. Balneario de Mondariz 1997.

Universidad Vigo. Master de Salud Natural. Ourense 1997.

Universidad de Valladolid. IV Curso: Estudio del dolor de espalda. Valladolid 1998.

Universidad de Sevilla. V Curso de puesta al día en el diagnóstico y tratamiento del dolor de espalda. Sevilla 1998.

Universidad de Cantabria. Curso de Especialización: Introducción a la Hidrología Médica. Santander 1998.

Universidad de Valladolid. V Curso: Estudio del dolor de espalda. Valladolid 1999.

Universidad de Sevilla. VI Curso de puesta al día en el diagnóstico y tratamiento del dolor de espalda. Sevilla 1999.

Universidad de La Coruña. Cursos de Doctorado - Bases clínicas de la Terapéutica Termal. Balneario de Lugo 1999.

Universidad de Salamanca. II Reunión de Estudios Históricos sobre Balnearios, Agua y Terapéutica. Los Balnearios Españoles. Salamanca 1999.

\section{Actividad Investigadora}

Como hemos tenido ocasión de señalar, desde su incorporación como alumna, a la Facultad de Medicina de la Universidad Complutense Josefina San Martín estuvo vinculada al laboratorio de la Cátedra de Hidrología Médica entonces en los locales de Farmacología. Tras el traslado de la Cátedra durante el curso 1970-71 a su actual ubicación en la $5^{\mathrm{a}}$ planta del Pabellón $5^{\circ}$ y alcanzado el acuerdo con el INDIME (Ministerio de Comercio: Dirección General de Comercio Interior. Servicio de Inspección de la Disciplina de Mercado) por el Prof. Armijo Valenzuela, se realizarón durante los años 1973 y 1974 el control de las aguas envasadas españolas ${ }^{2-3}$.

Josefina San Martín ya Médico Interno y Becaria de Investigación en virtud de concurso de méritos por la Dirección General de Universidades e investigación, desarrolló un papel relevante en las determinaciones analíticas microbiológicas de estos controles. 
No obstante, la propia Josefina San Martín en la "I Reunión de Estudios Históricos sobre Balnearios, Agua y Terapéutica" celebrada en Cestona (Guipúzcoa) en la primavera de 1996 indicaba:

"En estos últimos años hemos desarrollado un campo específico de investigación considerando las características propias de las explotaciones hidrotermales hasta llegar a su estado actual y asi se ha llevado a cabo un estudio histórico-científico de varios Establecimientos balnearios, algunos ya desaparecidos como Sacedón, u otros como Montemayor, Fuencaliente, Mondariz, Fuensanta, trabajos de investigación que han constituido temas de tesis doctorales, siendo destacables que dos de estos trabajos obtuvieron el Premio Internacional de Termalismo en 1993 y 1994.

Actualmente están en estudio los Establecimientos balnearios de Fortuna, Alhama de Aragón, Caldas de Oviedo, Arnedillo, etc. Y se están considerando la riqueza hidrotermal de determinadas zonas geográficas peninsulares.

Basándonos en el trabajo sobre los Balnearios y manantiales de la provincia de Teruel que incluyó además del análisis de las aguas un estudio histórico-científico de cada una de las aguas mineromedicinales, que con anterioridad habian sido utilizadas como agente terapéutico, y un plan de proyección futura, social y turística, y aprovechamiento posterior, hemos continuado el estudio de los manantiales de otras regiones y/o provincias españolas, tales como Álava, Soria, Pirineos, Madrid, siguiendo la misma metodología y enfoque en el estudio ${ }^{, 47}$.

Entre sus líneas de investigación destacan:

- "Acciones de las aguas mineromedicinales y minerales naturales en animales de investigación y en clínica

- Estudio histórico científico de los balnearios españoles

- Estudio analítico de las aguas mineromedicinales y minerales naturales

- Oligoelementos de las aguas mineromedicinales y minerales naturales

- Estudio de los microorganismos de las aguas

- Estudio de la población balnearia española" 48

Es coautora y coeditora de seis libros: Sauna (1976), La Salud por las Aguas Termales (1984), Hidroterapia (1986 a 1991) publicado en forma de fascículos en los Boletines de la Sociedad Española de Hidrología Médica desde el vol. I-1 al vol. VI- $1^{19-21}$ con la excepción de un número ${ }^{20}$, Medicina de Rehabilitación (1991), Rehabilitation Medicine (1991) y Curas Balnearias y Climáticas. Talasoterapia y Helioterapia (1994); y autora de veintisiete capítulos de libros. Teniendo publicados más de cien artículos en revistas científicas nacionales e internacionales (ver Anexo). Así mismo es autora de numerosos informes sanitarios vinculantes para la 
obtención de la declaración de aguas mineromedicinales y minerales naturales, sirvan de ejemplo entre las primeras las de: Baños de Benito (Albacete), El Trampal (Cáceres), Os Toxos (La Coruña), Baños de La Esperanza (Albacete), Baños de Vivaque (Toledo), Balneario Las Palmeras (Toledo), Sondeo Jafre (Gerona), Font de la Deu (Gerona) y Pou Samalús (Barcelona); y entre las segundas, las de: Font Ariant (Gerona), La Tayeda 3 (Gerona), Font d'Elka (Valencia), Font d'Arinsal (Andorra), Virgen del Camino (León), Pozo N. 2.899 Bixquert (Valencia), Font Sorda-Son Cocó (Baleares) y Font Mayor (Baleares) ${ }^{48}$.

Ha dirigido doce Tesis Doctorales (figura 7) y seis Tesinas, obteniendo todas las máximas calificaciones (ver Anexo).

Figura 7 - La doctora San Martín con su primer doctorando, Francisco Maraver, en Baños de Montemayor, verano de 1985

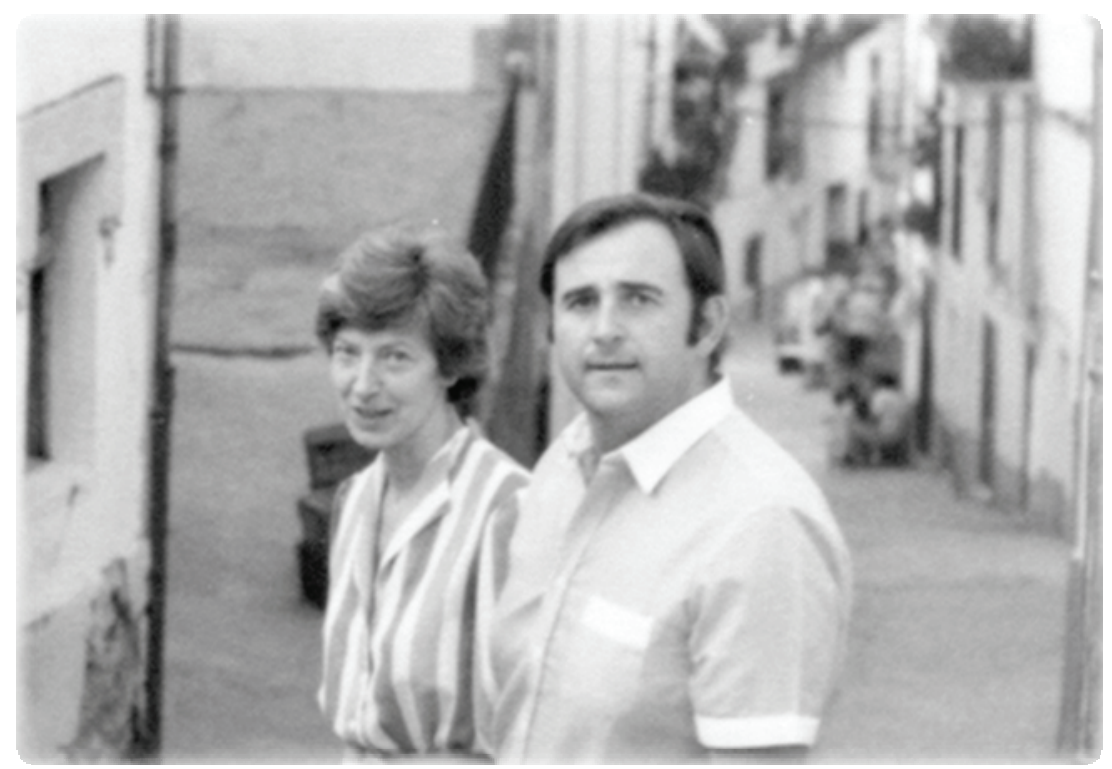

\section{Actividad Asociativa}

En la actualidad, la actividad asociativa vinculada al mundo de la Hidrología Médica en nuestro país, se identifica inexcusablemente con el dinamismo de la Sociedad Española de Hidrología Médica y de su órgano de expresión el Boletín. Desde su fundación por los médicos del Cuerpo de Baños en 1877, a partir de sus sesiones científicas e inicialmente en los Anales, ha canalizado la actividad científica de la especialidad ${ }^{30-31}$. 
Desde esta perspectiva, distinguimos dos etapas de estrecha vinculación de Josefina San Martín con la Sociedad: la primera como cercana colaboradora del Prof. Manuel Armijo, desde 1985 a 1991, y la segunda, ya como presidenta, desde 1991 a 1997.

Figura 8 - Portada de la revista Boletín de la Sociedad Española de Hidrología Médica
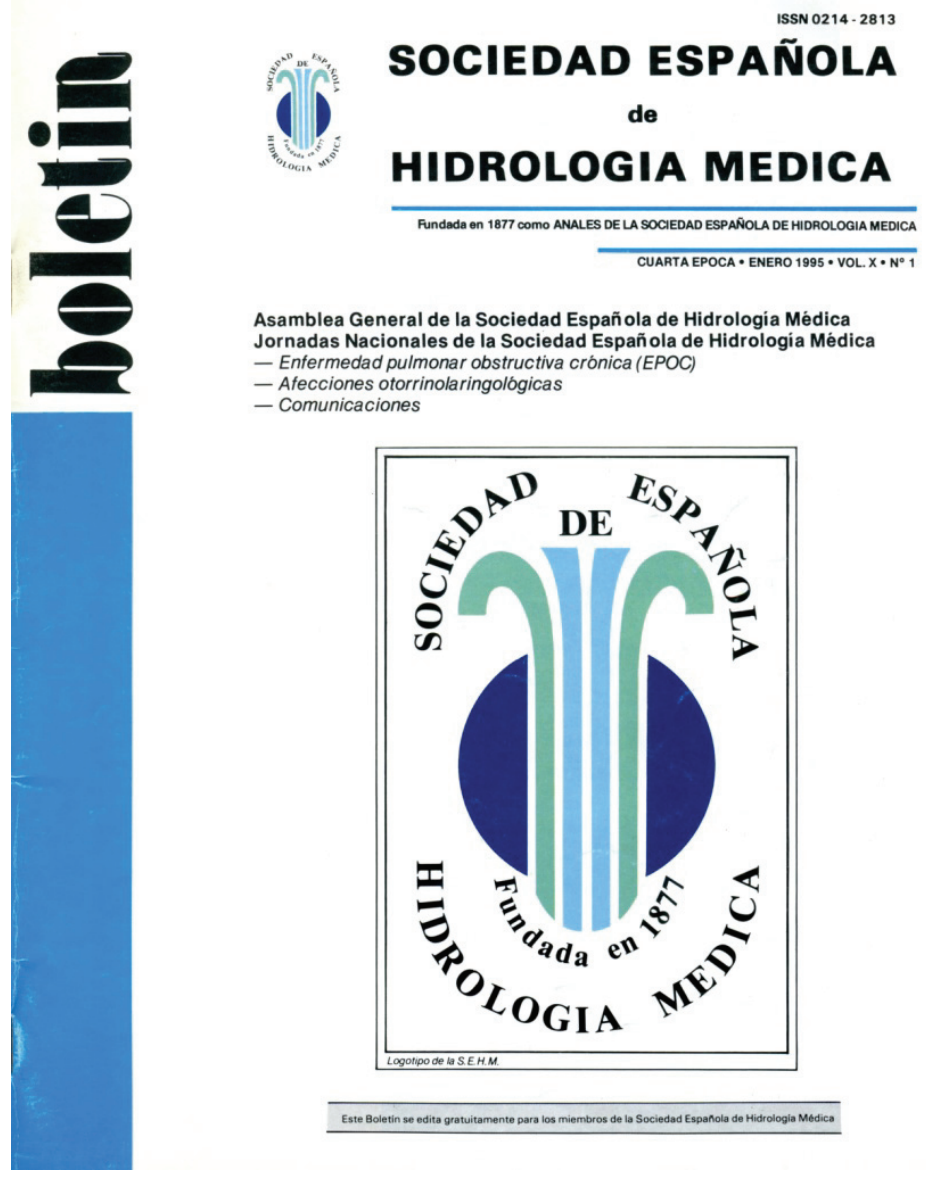

La Asamblea General celebrada el 24 de abril de 1985 designaba una nueva junta directiva en la que figuraban como presidente Manuel Armijo Valenzuela y como secretaria general Josefina San Martín Bacaicoa ${ }^{44-7}$. Uno de sus primeros objetivos fue volver a publicar el Boletín, en lo que constituía su cuarta época, lo que se logró en enero del año siguiente, figurando como Jefa de Redacción la doctora San Mar$\operatorname{tín}^{27}$. 
En este período destacan la celebración de dos Congresos. El Congreso Latino de Hidrología Médica, celebrado en el Balneario de Vichy Catalán de Caldas de Malavella del 20 a 22 de octubre de 1988 y del que se puede leer en el Boletín ${ }^{10}$ :

"Durante este Congreso y concretamente en la tarde del día 21, celebró la International Society of Medical Hydrology, una reunión bajo la presidencia del Prof. BOULANGE y a la que asistieron los presidentes de las Sociedades de Hidrología italiana, portuguesa, francesa y española, así como el Prof. RODRIGUEZ RODRIGUEZ. Presidente del "Congreso Europeo de Medicina Física y de Rehabilitación 89», a celebrar en Madrid del 5 al 9 de junio de este año. Se consideró la posibilidad de organizar el próximo congreso de la I.S.M.H. coincidiendo con el anteriormente citado de Medicina Física"

Así mismo, en el mismo número se señalaba':

"La Junta Directiva de la Sociedad Española de Hidrología Médica, en su reunión del pasado 2 de diciembre, consideró conveniente el prestar su más decidido apoyo a la organización y desarrollo de estos Congresos.

Presidente del Congreso Europeo de Medicina Física y de Rehabilitación es el Catedrático de Rehabilitación de la Universidad Complutense, Prof. Luís Pablo Rodriguez y del XXXI Congreso de la I.S.M.H. su Presidente, Prof. Michel BOULANGE. Catedrático de la Facultad de Medicina de Nancy (Francia)"

El día 9 de junio de 1989, en el Gran Anfiteatro Ramón y Cajal de la Facultad de Medicina de la Universidad Complutense de Madrid, se celebró una Sesión dedicada exclusivamente al XXXI Congreso Internacional de Hidrología y de Climatología Médica, el primer número del Boletín del año 1990 fue dedicado monográficamente al Congreso publicando los 21 resúmenes de conferencias, comunicaciones orales y carteles.

El 22 de abril de 1991, es elegida presidenta de la Sociedad en Junta General Ordinaria y desde esa fecha pasó a codirigir con el profesor Armijo el Boletín hasta su cese en ambos cargos el 12 de mayo de $1997^{49-23}$. Durante este periodo la Sociedad adquiere gran vitalidad, lo que se traduce en un aumento en el número de asociados ${ }^{12}$ y en múltiples iniciativas: apoyó la creación del premio internacional de termalismo para fomentar la labor realizada por los jóvenes investigadores en las distintas disciplinas relacionadas con el termalismo ${ }^{36}$; en el mismo sentido pero a

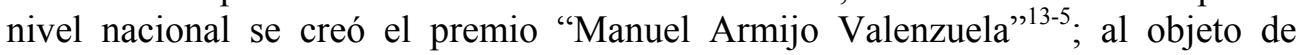
facilitar la adquisición de conocimientos de la especialidad, junto con el profesor Armijo y un grupo de colaboradores publicó el libro "Curas balnearias y Climáticas" $^{24}$; para modernizar el logotipo de la sociedad convocó un concurso que resuelto en la Asamblea correspondiente se publicó en la portada del Boletín ${ }^{25-13-5}$ (figura 8); renovó los estatutos y aprobó un reglamento electoral anexo a los mismos ${ }^{14}$; pero 
todo ello sin menoscabo de una amplia actividad científica, así en el último número del Boletín de 1996 podía leerse:

"Las reuniones a nivel nacional e internacional son trascendentes y con este criterio nuestra Sociedad ha pretendido, desde su fundación organizar este tipo de reuniones y así, en estos últimos diez años, se han celebrado Sesiones cientificas con temas selectivos tales como: "Crenoterapia en afecciones de vías urinarias" (septiembre de 1986); "El Termalismo en el envejecimiento fisiológico" (marzo de 1988); "Reumatismos No articulares" (diciembre 1990) y "La infección por el virus de la inmunodeficiencia humana" (noviembre de 1995). En alternancia con estas Sesiones Científicas, se han organizado Congresos y Jornadas, tales como: "Congreso Latino de Hidrología Médica" (Caldas de Malavella - octubre de 1988); "XXXI Congreso Internacional de Hidrología Médica y Climatología Médica" (MadridJunio de 1989); "Congreso de la S.E.H.M." (Hervideros de Cofrentes - Octubre, Noviembre de 1992); "Congreso de la S.E.H.M." (La Toja - Septiembre de 1993); "Jornadas Nacionales de la S.E.H.M." (Madrid-Noviembre de 1994); ....

...se hace especial referencia al próximo Congreso Internacional de nuestra sociedad, que se celebrará en el Balneario de Arnedillo (La Rioja), del 12 al 15 del presente año... “15

Por su importancia, quiero destacar su labor como presidenta del comité organizador de las I Jornadas de Aguas Minerales y Mineromedicinales celebradas en la Real Academia Nacional de Medicina, patrocinadas por el ITGE y los Ministerios de Industria, Comercio y Turismo y el de Obras públicas y Transporte ${ }^{48}$.

\section{Actividad Asistencial} rios $^{48}$ :

Desempeñó las Direcciones Médicas de los siguientes Establecimientos Balnea-

Balneario de Panticosa (Huesca). Temporada Oficial de 1977.

Balneario de Arnedillo (La Rioja). Temporadas Oficiales, de 1987 a 1999.

Balneario de Molgas (Orense). Temporada Oficial de 1981 (carácter interino).

San Juan de La Font Santa (Mallorca). Temporada Oficial 1985 (carácter interino).

Como alumno tuve la fortuna de recibir sus enseñanzas tanto en el Centro Nacional de Rehabilitación como en los balnearios de Arnedillo, Baños de Montemayor y Baños Molgas, pudiendo apreciar el cariño y respeto que le profesaban los enfermos con los que establecía rápidamente una relación de complicidad y empatía difícil de explicar. 


\section{Actividad Académica}

Con fecha 18 de Febrero de 1989 es nombrada Académica Correspondiente de la Real Academia Nacional de Medicina, pronunciando el 31 de octubre del mismo año, el discurso de recepción titulado "Acción diurética y ponderal de las aguas débilmente mineralizadas". Con posterioridad presentó, entre otras, las comunicaciones siguientes: "Talasoterapia: proyección terapéutica actual", "Interés actual de la talasoterapia en el estrés y cuadros asténicos", "Alteraciones psicosomáticas climatéricas y curas balnearias"...

Figura 9 - Marcial Campos y Josefina San Martín, en Karlovy Vary, invierno de $1987^{8}$

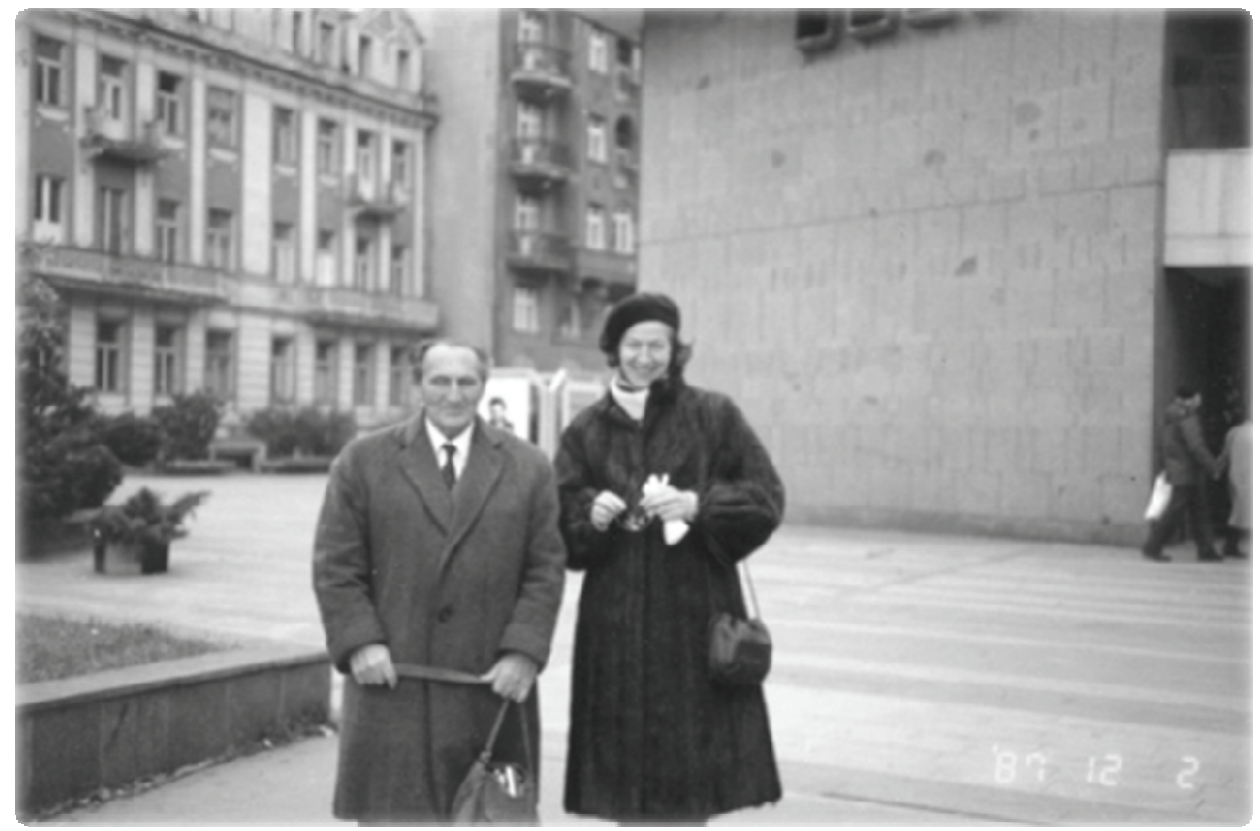

En 1993 fue nombrada miembro de la Comisión de Aguas Mineromedicinales de la Real Academia Nacional de Farmacia y el 19 de diciembre de 1996 Académica Correspondiente, pronunciando su discurso de ingreso el 10 de abril de 1997, con el título "Aguas mineromedicinales y minerales naturales como agentes medicamentosos".

Desde entonces ha presentado numerosas comunicaciones de diferentes aguas mineromedicinales de establecimientos balnearios españoles como las de: Carratraca (Málaga), Manzanera (Teruel), Jaraba (Zaragoza), Cervantes (Ciudad Real), 
Puente Viesgo (Cantabria), Valdelateja (Burgos), Alicún de las Torres (Granada), Villatoya (Albacete)...

Para finalizar este apartado, queremos destacar por su importancia, el discurso pronunciado el 25 de mayo de 1993, en la Real Academia de Ciencias Exactas, Físicas y Naturales, con el título: "La Hidrología Médica en España"48.

Figura 10 - Cartel de la Exposición 100 años haciendo ciencia

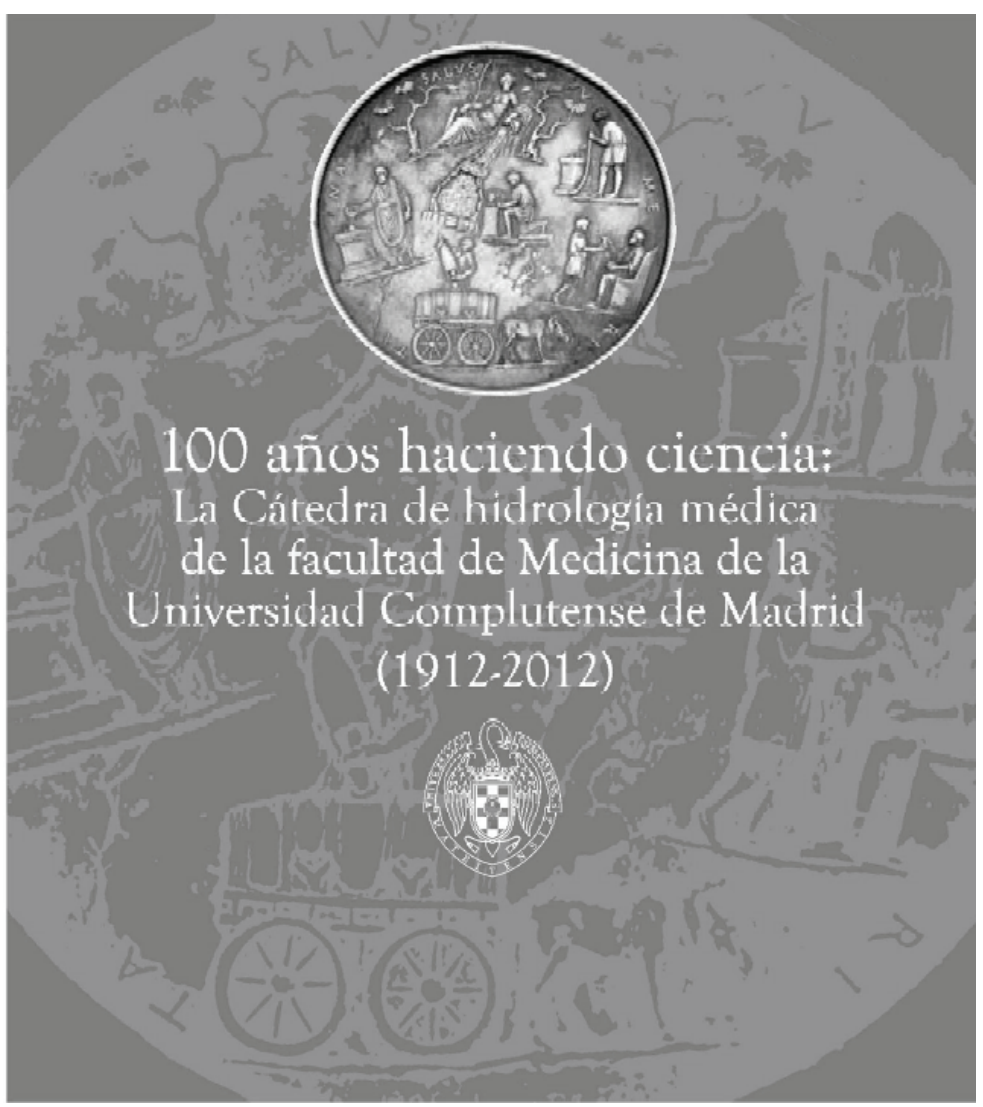

\section{A manera de colofón}

Teniendo en cuenta los méritos anteriormente citados, Josefina San Martín se convierte en una figura referente de la Hidrología Médica tanto a nivel nacional como internacional reconocida por todos, así en el año 2003 con motivo del primer número de la revista Thermaespa, su director Eloy Lozano le hizo una entrevista en la que ella manifestó sus opiniones sobre la Medicina termal: 
"Es positivo que una persona sin tener una patología determinada acuda al balneario para mejorar su condición de vida y su salud, pero siempre de un modo controlado" ó "El balneario no es sólo un lugar de mayores. También los niños pueden obtener grandes beneficios mediante tratamientos preventivos"

De ella el autor manifestó:

"Cercana y expresiva, llena de una energía que resulta entrañable, la entrevistamos en su cátedra de Madrid, rodeados de una historia que ella misma ha contribuido a crear ${ }^{, 26}$

Figura 11 - Lápida conmemorativa del centenario de la Cátedra

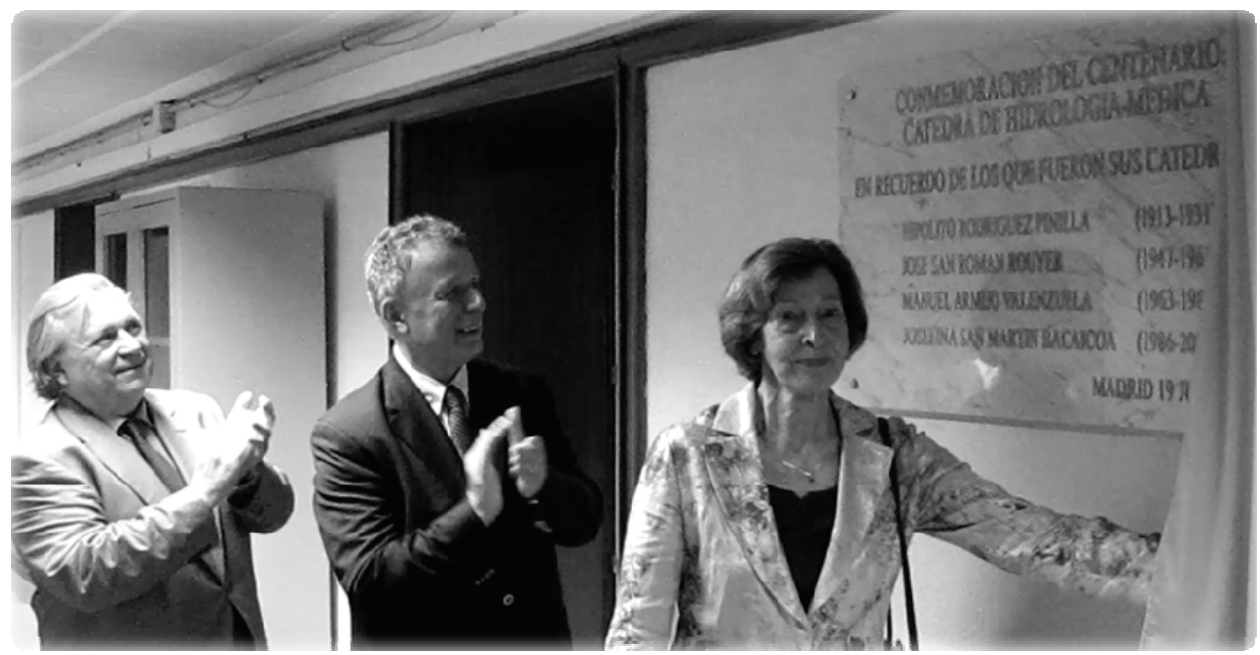

Por otra parte, desempeñó importantes cargos $^{48}$ relacionados con la especialidad:

Miembro de la Comisión Nacional de la Especialidad de Hidrología Médica a propuesta del Ministerio de Educación y Ciencia, desde 1985 y Presidenta desde 2000 a 2005.

Presidenta de la Sociedad Española de Hidrología Médica desde 1991 a 1997.

Vicepresidenta de la International Society of Medical Hydrology desde 1992 a1998 (No quiero dejar de señalar que de igual manera que en 1982 en Açores, el Prof. Manuel Armijo rechazara la propuesta de presidir la ISMH; el Prof. Boulangé ${ }^{6}$ subraya que Josefina San Martín declinó el mismo ofrecimiento, en 1991, con motivo del 31 Congreso celebrado en Madrid).

Presidenta del Consejo del Premio International de Termalismo desde 1991 a 1999. 
Miembro del Comité de Estudios del Consejo Nacional de Especialidades Médicas. Ministerio de Sanidad y Consumo y Ministerio de Educación, Cultura y Deporte. Desde 2001 a 2005.

Ha recibido numerosos premios y galardones ${ }^{11-39-48}$, entre los que destacan:

Premio Salgado de la Real Academia Nacional de Medicina.

Medalla de Oro del Termalismo Mundial, concedida por la Organización Mundial de Termalismo (OMTh) y la Sociedad Internacional de Técnica Hidrotermal (SITH) (figura 9).

Miembro de Honor de la International Society of Medical Hydrology.

Miembro de Honor de la Sociedad Portuguesa de Hidrología Médica.

Medalla de la Asociación Gallega de la Propiedad Balnearia.

Josefina San Martín es una protagonista fundamental en el devenir de la Cátedra $^{32-40-41-42}$. Así desde el punto de vista de la docencia, gracias a la Ley de 20 de julio de 1955 sobre especialidades médicas que posibilitó la creación de la Escuela Profesional, lo que inicialmente sólo fue asignatura libre del doctorado, incorporó el programa vigente de la especialidad de $1988^{18-28-35-46-52}$; y ya desde 1994, asignaturas optativas de las licenciaturas de Medicina y Ciencias y Tecnología de los Alimentos y de la Diplomatura de Fisioterapia.

Para finalizar, quiero destacar su implicación como responsable de las actividades realizadas en el presente ejercicio con motivo de la celebración del centenario de la Cátedra de Hidrología Médica.

Coordinó el acto académico celebrado el 17 de abril de 2012 en la Sala de Grados de nuestra Facultad de Medicina, siendo la principal promotora de la exposición* que con el título "Cien años haciendo Ciencia"43-50 (figura 10) se inauguró el mismo día permaneciendo abierta hasta el 19 de junio, en que descubrió una lápida conmemorativa en su Cátedra (figuras 11 y12).

(*) La exposición utilizó como motivo la pátera de Otañes, concretamente la medalla conmemorativa realizada por la $\operatorname{Prof}^{\mathrm{a}}$ Consuelo de la Cuadra con motivo del I Congreso Peninsular de Termalismo Antiguo ${ }^{1}$. El contenido está disponible en: http://www.ucm.es/BUCM/med/51068.php ${ }^{43}$

\section{Agradecimientos}

Quiero agradecer a la profesora Josefina San Martín su generosa colaboración para la realización de esta breve semblanza, especialmente por las aclaraciones de su iconografía y currículo en la cordial reunión mantenida el día 13 diciembre de 2012 que han enriquecido y facilitado mi labor. También a la doctora $\mathrm{M}^{\mathrm{a}}$ Ángeles Ceballos por la información ofrecida en su presentación con motivo del Acto Académico de Conmemoración del centenario de la creación de la Cátedra de Hidrología Médica celebrado en la Sala de Grados de la Facultad de Medicina de la UCM el día 17 de abril de 2012. 
Figura 12 - Profesores San Martín y Maraver reunidos en diciembre 2012

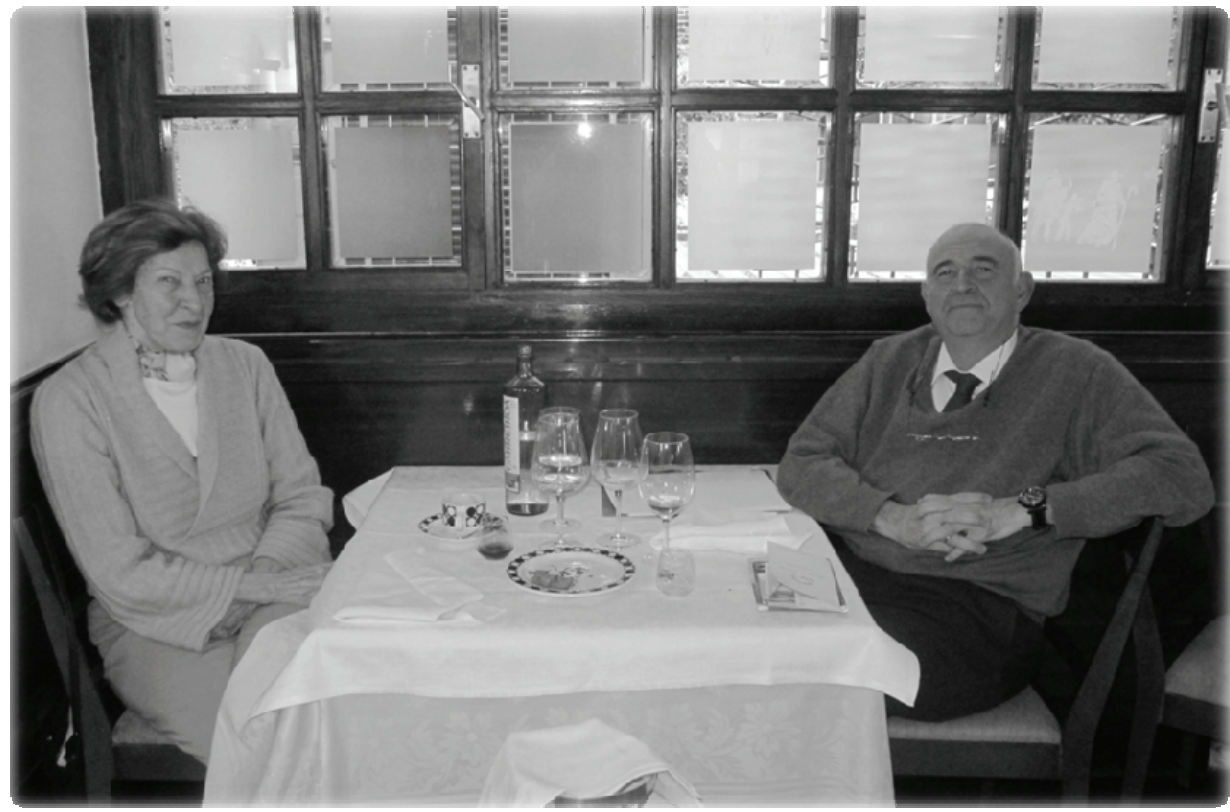

\section{BIBLIOGRAFÍA}

1. Abad M. Medalla conmemorativa del I Congreso Peninsular de Termalismo Antiguo. In: Peréx MJ (Ed.). Termalismo Antiguo. Madrid: UNED-Casa de Velázquez, 1997: 573-574.

2. Armijo F. Los análisis de aguas en la Cátedra de Hidrología Médica. Acto Académico de Conmemoración del centenario de la creación de la Cátedra de Hidrología Médica, 2012 Abr 17; Madrid UCM, 2012 [consultado 17-052012]: Disponible en: http://www.ucm.es/BUCM/med/doc19733.pdf

3. Armijo F. Manuel Armijo Valenzuela. Balnea 2012; 7: 95-134.

4. Armijo M. Antecedentes históricos y evolución de la Especialidad. In: Armijo M et al. Hidrología. Madrid: Organización Médica Colegial, 1990: 1-15.

5. Asamblea General Ordinaria. Bol Soc Esp Hidrol Med 1995; 10(1): 7.

6. Boulangé M. La présense française au sein de l'ISMH. In: Jeambrun P (dir.) Florílège Thermal. Les 150 ans de la Société. Paris: Sociétè Française d'Hydrologie et de Climatologie Médicales, 2006: 19-26. 
7. Ceballos MA, San José JC. La Sociedad Científica y sus actividades. In: Armijo M et al. Hidrología. Madrid: Organización Médica Colegial, 1990: 3342.

8. Ceballos MA. Ilma. Sra. Dña. Josefina San Martín Bacaicoa (1986-2004). Acto Académico de Conmemoración del centenario de la creación de la Cátedra de Hidrología Médica, 2012 Abr 17; Madrid UCM, 2012 [consultado 1705-2012]: Disponible en: http://www.ucm.es/BUCM/med/doc19732.pdf

9. Congreso Europeo de Medicina Física y Rehabilitación y XXXI Congreso de la International Society of Medical Hydrology and Climatology. Bol Soc Esp Hidrol Med 1989; 4(1): 6.

10. Congreso Latino de Hidrología Médica. Bol Soc Esp Hidrol Med 1989; 4(1): 5-6.

11. Concesión. Bol Soc Esp Hidrol Med 2005; 20(1): 58.

12. Directorio de Asociados de la Sociedad Española de Hidrología Médica. Bol Soc Esp Hidrol Med 1992; 7(3): 158-162.

13. Editorial. Bol Soc Esp Hidrol Med 1995; 10(1): 5-6.

14. Editorial. Bol Soc Esp Hidrol Med 1996; 11(2): 65.

15. Editorial. Bol Soc Esp Hidrol Med 1996; 11(3): 109-110

16. Etayo J, Galino F, Portela F. Universidad Complutense de Madrid de la Edad Media al III Milenio. Madrid: Universidad Complutense de Madrid, 2002.

17. Galino F. Del Protocolo y Ceremonial Universitario Complutense. Madrid: Universidad Complutense de Madrid, 1999.

18. Hidrología. BOE 1988; 220: 27074-27076.

19. Hidroterapia. Bol Soc Esp Hidrol Med 1986; I(1): 4.

20. Hidroterapia. Bol Soc Esp Hidrol Med 1989; IV(3): 99.

21. Hidroterapia. Bol Soc Esp Hidrol Med 1991; 6(1): 5.

22. Informaciones, avisos y noticias. Nombramientos. Bol Soc Esp Hidrol Med 1987; II(1): 42.

23. Junta General Ordinaria. Bol Soc Esp Hidrol Med 1997; XII(3): 129-130.

24. Libros - Curas Balnearias y Climáticas. Talasoterapia. Helioterapia. Bol Soc Esp Hidrol Med 1994; 9(3): 175.

25. Logotipo de la Sociedad Española de Hidrología Médica. Bol Soc Esp Hidrol Med 1994; 9(3): 172.

26. Lozano E. Josefina San Martín Bacaicoa. Una vida dedicada a la medicina del agua. Thermaespa 2003; 1: 78-84.

27. Maraver F, Corvillo I. La Publicación Científica especializada. In: Armijo M et al. Hidrología. Madrid: Organización Médica Colegial, 1990: 45-55.

28. Maraver F, Aguilera L, Meijide R, Saz P. Academic implememtation of health resort medicine (classical natural medicine and medical hydrology and climatology) in spanish universities. Proceedings of the 33rd World Congres International Socity of Medical Hydrology and Climatology; 1998 Oct 4-11; Karlovy Vary, 1998: 213. 
29. Maraver F, Corvillo I, Aguilera L, Armijo F. 25 années de l'Ecole d'Hydrologie Médicale et d'Hydrothérapie de Madrid. Proceedings of the VII Congres Transpyreneen de Medecine Physique \& de Readaptation; 2003 Nov 21-22; Toulouse, 2003: 207.

30. Maraver F, Corvillo I. Historia de la Sociedad Española de Hidrología Médica. Siglo XIX. Balnea 2006; 2: 274 pp.

31. Maraver F. L'hydrologie médicale dans l'Espagne de "La Restauration": 1874 - 1902. Press Therm Climat 2007; 144: 151-163.

32. Maraver F, Aguilera L, Corvillo I, Morer C, Armijo F. L'Enseignement de l'Hidrologie à l'Univerdité Complutense de Madrid. Trente années de l'Ecole professionnelle d'Hydrologie Médicale et d'Hydrothérapie. Press Therm Climat 2007; 144: 167-176.

33. Maraver F, Álvarez-Badillo A, Gómez F, Romero M, Meijide R, Armijo F. Propuesta de programa de la especialidad de "Hidrología Médica". Anal Hidrol Med 2010; 3: 157-206.

34. Maraver F. La Cátedra de Hidrología Médica y la Escuela Profesional de Hidrología Médica e Hidroterapia. Acto Académico de Conmemoración del centenario de la creación de la Cátedra de Hidrología Médica, 2012 Abr 17; Madrid Universidad Complutense, 2012 [consultado 17-05-2012]: Disponible en: http://www.ucm.es/BUCM/med/doc19735.pdf

35. Nogales A. Presentación. An Hidrol Med 2006; 1: 7.

36. Premio Internacional de Termalismo. Bol Soc Esp Hidrol Med 1992; 7(2): 114

37. Rodríguez-Rodríguez LP. Excmo. Sr. D. Manuel Armijo Valenzuela (19631986). Acto Académico de Conmemoración del centenario de la creación de la Cátedra de Hidrología Médica, 2012 Abr 17; Madrid Universidad Complutense, 2012.

38. Rodríguez-Rodríguez LP. Discurso de precepto, en nombre de la Academia, a cargo del Excmo. Sr. D. ... . Solemne Sesión Necrológica en memoria del Excmo. Sr. D. Manuel Armijo Valenzuela 19 de junio de 2012. An R Acad Nac Med (Madr) 2012; (en prensa).

39. Rodríguez-Sánchez JA. José Salgado y Guillermo: el Premio en la Historia. Bol Soc Esp Hidrol Med 1994; 9: 105-111.

40. Rodríguez-Sánchez JA. La creación de la Cátedra de Hidrología Médica (1912). In: Castellanos J, Jiménez I, Ruíz MJ, Gardeta P (coord.). La medicina en el siglo XX. Estudios Históricos sobre Medicina, Sociedad y Estado. Málaga: Sociedad Española de Historia de la Medicina, 1998: 201-212.

41. Rodriguez-Sanchez JA. Antecedentes históricos: las memorias del agua. In Baeza J, López JA, Ramírez A, (ed.). Las aguas minerales en España. Madrid: IGME, 2001:1-15.

42. Rodríguez-Sánchez JA. Institucionalización de la Hidrología Médica en España. In: Maraver F (coord.). Establecimientos balnearios: historia, literatura y medicina. Madrid: Universidad Complutense de Madrid, 2006: 25-40. 
43. Rodríguez-Sánchez JA, De Jorge J, Crego M. Cien años haciendo ciencia: la Cátedra de Hidrología Médica de la Faculta de Medicina de la Universidad Complutense (1912-2012). Catálogo de la exposición. Balnea 2012; 7: 263308. Exposición virtual [consultado 17-04-2012]: disponible en: http://www.ucm.es/BUCM/med/51068.php

44. San José JC. Sociedad Española de Hidrología Médica (datos y fechas). Bol Soc Esp Hidrol Med 1986; I(1): 4-10.

45. San Martín J, Armijo M. La formación de especialistas en las Comunidades Europeas. In: Armijo M et al. Hidrología. Madrid: Organización Médica Colegial, 1990: 73-87.

46. San Martín J. Health Resort Medicine. Integration in Universities. In: Pratzel HG (ed.). Health Resort Medicine. München: ISMH, 1995: 45-48.

47. San Martín J, Martínez I. Investigación hidrológica en la Cátedra de Madrid. Apuntes sobre un trabajo en curso. In: Urkía JM \& Rodríguez-Sánchez JA (coords.). Los Balnearios Españoles. Salamanca: ANET, 1998: 159-168.

48. San Martín MMJ. Curriculum vitae María Magdalena Josefina San Martín Bacaicoa para el nombramiento de Profesor Emérito, 2004 Mar 20; Madrid Universidad Complutense, 2012 [consultado 17-04-2012]: Disponible en: http://www.ucm.es/BUCM/med/doc19524.pdf

49. Sociedad Española de Hidrología Médica. Bol Soc Esp Hidrol Med 1991; 6(2): 59-60.

50. Suarez M. Conmemoración del I centenario de la creación de la Cátedra de Hidrología Médica de la universidad española (1912-2012), 2012 Nov 23; Termas World Agency [consultado 30-11-2012]: Disponible en: http://termasworld.com/content/view/363/45/

51. Symposium: Medical Hydrology in Rehabilitation. Bol Soc Esp Hidrol Med 1990; V(3): 123-129.

52. VVAA. Hidrología. In: Guía de formación de Médicos especialistas. Madrid: Ministerio de Sanidad y Consumo, 1986: 371-378.

\section{ANEXO}

Producción científica más relevante de la doctora Josefina San Martín Bacaicoa: publicaciones en revistas científicas nacionales e internacionales, libros y capítulos de libros, aportaciones a congresos nacionales e internacionales y relación de tesis y tesinas dirigidas. Cerca de trescientas anotaciones fruto de una dilatada labor profesional y científica. 


\section{PUBLICACIONES}

\section{Publicaciones en revistas científicas}

Nacionales

1. Armijo M, Rodríguez LP, Rodríguez JA, San Martín J. Acción del agua radiactiva sobre el crecimiento de cultivos de corazón embrionario. Arch Fac Med 1972; 22(4): 185-189

2. San Martín J. Aguas Naturales. Med Moderno 1972; VII: 33-36

3. Velasco A, Arévalo JM, San Martín J, Armijo M. Efectos de algunos psicofármacos sobre el consumo de oxígeno y glucosa en cerebro de Rata "in vitro". Rev Esp Fisiol 1973; 29(1): 47-50

4. Gutierrez C, Desviat M, San Martín J, Ruiz D. El dolor en los hemipléjicos. Rehabilitación 1973; 7(4): 501-505

5. Armijo M, San Martín J, Armijo F. Acciones de la sauna en sujetos normales. Rehabilitación 1974; 8(3): 559-575

6. Velasco-Martín JL, Arévalo JM, San Martín J, Velasco-Martín A. Efectos del citrato de propaxolina sobre el Consumo de oxígeno en algunos tejidos de Ratas "in vitro". Acta Reumatol 1974; 1: 41-46

7. Armijo M, San Martín J, Armijo F. Contenido en ión fluoruro de algunas Aguas Minero-Medicinales utilizadas frecuentemente como aguas de mesa. Anal Bromatol 1976; 28(2): 179-192

8. Rodán A, San Martín J. Rehabilitación en vasculopatías venosas Periféricas. Rehabilitación 1978; 12(1): 135-142

9. Armijo F, San Martín J, Armijo M. Representación gráfica del análisis de Aguas Minero-Medicinales. Anal Bromatol 1978; 30(1): 90-96

10. San Martín J, Rodán A. Comparación del eterilato y del ácido acetilsalicílico en el tratamiento de Cervicoartrosis: prueba doble ciego. Arch Farm Toxicol 1978; 4(1): 41-46

11. Armijo F, San Martín J, Armijo M. Contenido en sílice de algunas Aguas Minerales. Anal Bromatol 1979; 31(3-4): 365-373

12. Rodán A, Desviat M, Prada S, García V, Osés J, San Martín J, Pascual T. Rehabilitación en cirugía ortopédica de la Cadera. Rehabilitación 1979; 13(2): 135-162 
13. Armijo M, San Martín J. Hidroterapia y Crenoterapia en el envejecimiento osteoarticular. Reumatol Práct 1980; 4(4): 109-118

14. San Martín J, Rodán A. Hidroterapia en cervicalgias psicógenas. Rehabilitación 1980; 14(3): 301-306

15. Armijo F, San Martín J, Armijo M. Contenido en Magnesio y relación calciomagnesio en algunas Aguas Minero-Medicinales envasadas. Anal Bromatol 1980; 32(3): 215-222

16. Armijo M, San Martín J. Hidroterapia y Crenoterapia en la Gonartrosis. Rheuma 1982; 1: 73-78

17. Armijo M, San Martín J. Hidroterapia y Crenoterapia en el tratamiento de la inflamación reumática crónica. Rheuma 1982; Extra: 82-90

18. Armijo M, San Martín J. Hidroterapia y Crenoterapia en la coxartrosis. Rheuma 1982; 2: 63-69

19. Armijo M, San Martín J. Hidroterapia y Crenoterapia en las lumboartrosis. Rheuma 1982; 3: 58-64z

20. San Martín J, Armijo F, Armijo M. Efectos de los baños de sauna sobre el equilibrio catiónico en sudor, plasma y orina de sujetos normales. Rehabilitación 1983; 17(2): 165-174

21. Armijo M, San Martín J. La cura hidrotermal en las algias reumáticas. Rheuma 1983; 7(1): 56-60

22. Armijo M, San Martín J. Hidroterapia y Crenoterapia en la Artritis Reumatoide. Rheuma 1984; 8(1): 55-60

23. San Martín J, Armijo M. Hidroterapia y Crenoterapia en los reumatismos yuxtaarticulares. Rheuma 1985; 22: 52-58

24. San Martín J, San Martín I. Algodistrofias: Crenoterapia e Hidroterapia. Bol Soc Esp Hidrol Med 1986; 1(1): 21-25

25. Fuentes A, San Martín J. Acción de la Creno-Helio-Talasoterapia en el tratamiento de la Psoriasis. Bol Soc Esp Hidrol Med 1986; 1(1): 27-30

26. San Martín J. Actualidad del Termalismo. Bol Soc Esp Hidrol Med 1986; 1(2): 53-56

27. San Martín J. Crenoterapia en las afecciones renales y vías urinarias. Bol Soc Esp Hidrol Med 1986; 1(3): 101-103

28. San Martín J, Armijo F. Balnearios y manantiales de aguas minerales de la provincia de Teruel. Estudio histórico-científico y proyección social y turística. Teruel - CSIC 1986; 75(1): 49-94 
29. San Martín J. Las Curas balnearias en Geriatría. Bol Soc Esp Hidrol Med 1987; 2(3): $33-37$

30. Maraver F, Crespo PV, Sanchez-Quevedo MC, Campos A, San Martín J. Microscopia electrónica del material orgánico de las aguas sulfuradas. Bol Soc Esp Hidrol Med 1987; 2(3): 133-135

31. San Martín J. Reumatismos psicógenos y curas balnearias. Bol Soc Esp Hidrol Med 1988; 3(1): 35-38

32. Armijo F, Maraver F, San Martín J. Análisis de las aguas de Montemayor. An Bromatol 1988; 40: 147-153

33. San Martín J. La cura termal en el Establecimiento Balneario de Arnedillo: Factores terapéuticos, indicaciones y contraindicaciones, técnicas y resultados. An Real Acad Farm 1988; 14: 33-46

34. San Martín J, San José MC. Paso a través de la piel de los factores mineralizantes de las aguas utilizadas en Balneación. Bol Soc Esp Hidrol Med 1989; 4(1): 27-32

35. Asirón PJ, San Martín J. Balneotermoterapia. Bol Soc Esp Hidrol Med 1989; 4(2): $55-58$

36. San Martín J. Crenoterapia en afecciones respiratorias. Bol Soc Esp Hidrol Med 1989; 4(3): 133-136

37. San Martín J. Acción diurética y ponderal de las aguas débilmente mineralizadas. An R Acad Nac Med (Madr) 1989; 106(4): 567-583

38. San Martín J. Termalismo tradicional - Turismo Termal -Turismo de Salud. Bol Soc Esp Hidrol Med 1990; 5(2): 83-86

39. San Martín J. Evaluación de la utilidad de la cura balnearia. Bol Soc Esp Hidrol Med 1991; 6(1): 9-11

40. San Martín J. Consideraciones en torno a las técnicas Rehabilitadoras de la coxartrosis en el agua. Bol Soc Esp Hidrol Med 1991; 6(2): 79-88

41. San Martín J. Crenocinesiterapia e Hidrocinesiterapia en las afecciones de rodilla. Bol Soc Esp Hidrol Med 1991; 6(3): 125-130

42. San Martín J. Hidroterapia y Crenoterapia en las afecciones del pie. Bol Soc Esp Hidrol Med 1992; 7(1): 29-35

43. Ceballos MA, San Martín J. El Médico del agua. Bol Soc Esp Hidrol Med 1992; 7(1): 49-52

44. San Martín J, Ceballos MA. Extremidad superior, funcionalidad y patología: Hidroterapia y Crenoterapia. Bol Soc Esp Hidrol Med 1992; 7(2): 67-76 
45. San Martín J. Piscinas de tratamiento. Higiene y control. Bol Soc Esp Hidrol Med 1993; 8(2): 102-105

46. San Martín J. Relaciones entre Balneoterapia y Rehabilitación. Bol Soc Esp Hidrol Med 1993; 8(3): 129-133

47. San Martín J. La Hidrología Médica en España Contribución académica al conocimiento y aprovechamiento de las aguas subterráneas en España. Revista de la Real Academia de Ciencias Exactas, Físicas y Naturales 1994; 88(1): 8590

48. Nappi G, Calcaterra P, Masciocchi MM, De Luca S, San Martín J. Estudio experimental de la acción de las aguas bicarbonatadas cálcicas de Sangemini (Italia) en síndromes dispépticos de origen gástrico. Bol Soc Esp Hidrol Med 1994; 9(1): 15-20

49. Martínez I, Armijo F, San Martín J. Los nitratos en las aguas. Situación actual de las aguas envasadas españolas. Bol Soc Esp Hidrol Med 1994; 9(1): 27-34

50. San Martín J. Osteoporosis involutiva y Curas Balnearias. Bol Soc Esp Hidrol Med 1994; 9(3): 127-131

51. Armijo F, San Martín J. Conductividad eléctrica de las Aguas MineroMedicinales. Bol Soc Esp Hidrol Med 1994; 9(3): 157-162

52. San Martín J. Talasoterapia: Proyección terapéutica actual. An R Acad Nac Med (Madr). 1995; 112(2):347-379.

53. Nappi G, De Luca S, Fortunati NA, Masciocchi MM, San Martín J. Tratamiento termal en las hipoacusias rinógenas por insuflación de gas. Bol Soc Esp Hidrol Med 1995; 10(2): 75-78

54. Arias JL, Armijo F, San Martín J. Concepto de Termalidad y Aguas Minerales. Bol Soc Esp Hidrol Med 1995; 10(2): 93-100

55. San Martín J. Manganeso en las aguas minerales. Bol Soc Esp Hidrol Med 1995; 10(3): 139-144

56. San Martín J. Interés actual de la Talasoterapia en el estrés y cuadros asténicos. An R Acad Nac Med (Madr) 1996; 113(2): 457-469

57. San Martín J. Consideraciones en torno a las psicalgias y curas balnearias. Bol Soc Esp Hidrol Med 1996; 11(1): 9-16

58. San Martín J. Influencia psicoterápica del médico en las Curas Balnearias. Bol Soc Esp Hidrol Med 1996; 11(3): 111-117

59. San Martín J. Alteraciones psicosomáticas en el climaterio femenino y curas balnearias. An R Acad Nac Med (Madr) 1997; 114(1): 129-42 
60. San Martín J. Aguas mineromedicinales y minerales naturales como agentes medicamentosos. An Real Acad Farm 1997; 63(4): 777-800

61. López A, San Martín J, Martínez I, Armijo F, San José JC. Estudio comparativo-analítico de las aguas mineromedicinales del Balneario Fortuna-Leana. Bol Soc Esp Hidrol Med 1999; 14(3): 119-132

62. San Martín J, Castejón A. Acciones terapéuticas de las aguas del Balneario de Carratraca. (Memoria no 23). An Real Acad Farm 1999; 65(E): 535-553

63. San Martín J, Valero A. Acción terapéutica de las aguas del Balneario "El Paraíso" de Manzanera (Teruel) (Memoria $n^{\circ}$ 24). An Real Acad Farm 2001; 67(E): 235-551

64. Arribas AM, Elizalde I, Zubia AR, San Martín J. Revisión de los conocimientos actuales sobre microelementos minerales u oligoelementos. Bol Soc Esp Hidrol Med 2001; 16(2): 67-69

65. Zubia AR, Elizalde I, San Emeterio MM, San Martín J. Oligoelementos esenciales: biodisponibilidad y papel fisiológico. Bol Soc Esp Hidrol Med 2001; 16(2): 71-82

66. Zubia AR, Ortega L, Arribas AM, San Martín J. Hierro - oligoelemento esencial. Bol Soc Esp Hidrol Med 2001; 16(2): 83-90

67. Carmona E, Ortega L, San Emeterio MM, San Martín J. Cobre - oligoelemento esencial. Bol Soc Esp Hidrol Med 2001; 16(2): 91.98

68. San Emeterio MM, Carmona E, Ortega L, San Martín J. Selenio - oligoelemento esencial. Bol Soc Esp Hidrol Med 2001; 16(2): 99-101

69. Elizalde I, Zubia AR, Arribas AM, San Martín J. Resumen sinóptico de los Oligoelementos Esenciales en humanos. Legislación y recomendaciones dietéticas. Bol Soc Esp Hidrol Med 2001; 16(3): 127-134

70. Ortega L, Carmona E, Elizalde I, San Martín J. Zinc - Oligoelemento Esencial. Bol Soc Esp Hidrol Med 2001; 16(3): 135-141

71. Ortega L, Arribas AM, Zubia AR, San Martín J. Manganeso - Oligoelemento Esencial. Bol Soc Esp Hidrol Med 2001; 16(3): 143-146

72. San Martín J, Valero A. Acción terapéutica de las aguas del Balneario de "Alhama de Granada". (Memoria $n^{\circ}$ 25). An Real Acad Farm 2002; 68(E): 154-185

73. Romero M, Serrano C, San Martín J. Epidemiología de la legionelosis y consideraciones sobre su prevención en balnearios. Bol Soc Esp Hidrol Med 2002; 17(2): $67-89$ 
74. San Martín J, Perea MA. El Olimpo y el Mar. Bol Soc Esp Hidrol Med 2003; 18(2): $67-76$

75. Navarro E, Alonso SJ, San Martín J. Resumen histórico farmacológico terapéutico de las aguas minerales de Firgas. Canar méd quir 2003; 1(1): 50-53

76. Navarro E, Alonso SJ, San Martín J. Constancia diacrónica de las aguas minerales de Firgas (Islas Canarias) y usos terapéuticos. Nutr clín diet hosp 2003; 23(3): $15-23$

77. San Martín J, Valero A. Acción terapéutica de las aguas de los Balnearios de Jaraba. (Memoria $n^{\circ}$ 26). An Real Acad Farm 2004; 70(E): 625-653

78. Navarro E, Alonso SJ, Hernández F, San Martín J. Importancia para la salud de las aguas minerales magnésicas. Aguas minerales de Firgas (Islas Canarias). Nutr clín diet hosp 2005; 25(2): 28-38

79. San Martín J. Patología psicosomática y cura balnearia. Bol Soc Esp Hidrol Med 2005; 20(2): 6-21

80. San Martín J, Valero A. Acción terapéutica de las aguas del Balneario Cervantes. (Memoria n ${ }^{\circ}$ 27). An Real Acad Farm 2006; 72(E): 399-431

81. Navarro E, Alonso SJ, Hernández F, San Martín J. Las aguas mineromedicinales del Pozo de la Salud. El Hierro. Islas Canarias. Nutr clín diet hosp 2006; 26(2): 9-23

82. Navarro E, Hernández F, Alonso SJ, San Martín J. Cuantificación de la actividad laxante de las aguas del Pozo de la Salud (El Hierro, Islas Canarias). Bol Soc Esp Hidrol Med 2007; 22(2): 17-21

83. Navarro E, Alonso SJ, San Martín J. Screening farmacológico preliminar de las aguas de San Antón (Gran Canaria, Islas Canarias). Bol Soc Esp Hidrol Med 2007; 22(2): 22-27

84. Navarro E, Velázquez R, Alonso SJ, San Martín J. Actividad conductual y neurológica de las aguas minero-medicinales de Teror (Gran Canaria, Islas Canarias). Bol Soc Esp Hidrol Med 2007; 22(2): 29-34

85. San Martín J, Valero A. Acción terapéutica de las aguas del Balneario de Puente Viesgo. (Memoria no 28). An Real Acad Farm 2007; 73(E): 361-389

86. Navarro E, Alonso SJ, San Martín J. Climatoterapia de la cuenca del barranco de La Virgen (Islas Canarias). An Hidrol Med 2007; 2: 27-35

87. Navarro E, Alonso SJ, San Martín J. Efectos neurológicos y hepatoprotectores de las aguas minerales de Firgas (Islas Canarias).An Hidrol Med 2007; 2: 37 45 
88. San Martín J, Valero A. Las aguas del Balneario de Valdelateja (Burgos). Posibles acciones e indicaciones según vía de administración. Programas ofrecidos y estudio analítico de los usuarios. (Memoria $n^{\circ} 29$ ). An Real Acad Farm 2008; 74(E): 609-623

89. San Martín J, Valero A. Estudio de la acción terapéutica de las aguas del Balneario de Alicún de las Torres (Granada). (Memoria $n^{0}$ 30). An Real Acad Farm 2009; 75(E): 849-870

90. Navarro E, Velázquez R, San Martín J. Actividad estimulante intestinal de las aguas minerales de Teror (Gran Canaria, Islas Canarias). Bol Soc Esp Hidrol Med 2010; 25(2): 67-70

91. Navarro E, Velázquez R, San Martín J. Influencia del pH del agua mineral de Firgas sobre la excreción urinaria de salicilato sódico (Gran Canaria, Islas Canarias). Bol Soc Esp Hidrol Med 2010; 25(2): 70-72

92. San Martín J, Valero A. Estudio de la acción terapéutica de las aguas del Balneario Baños de la Concepción de Villatoya (Albacete). (Memoria $\mathrm{n}^{\mathrm{o}} 31$ ). An Real Acad Farm 2011; 77(E): 149-179

Internacionales

1. Armijo M, San Martín J, Armijo F. Relaciones catiónicas de las aguas cloruradas marinas o terrestres. Med Term Clim 1984; 62: 5-9

2. Armijo M, San Martín J. Talasoterapia en la Tercera Edad. Med Term Clim 1984; 63: 13-16

3. San Martín J. Crenoterapia de los reumatismos. Publ Inst Clim Hidrol Univ Coimbra 1985; 28: 115-122

4. San Martín J. Crenoterapia de los reumatismos. Arq reumatol doenças ósteoarticul 1986: 8(7): 273-280

5. Corvillo I, Armijo F, San Martín J. Acción de los aeroiones sobre gérmenes ambientales. Publ Inst Clim Hidrol Univ Coimbra 1987; 29: 51-53

6. Alvaro-Gracia JM, San Martín J, Alvaro-Gracia A. Ionización negativa en la recuperación del deportista. Publ Inst Clim Hidrol Univ Coimbra 1987; 29: 5557

7. García I, San Martín J. Hidroterapia en el tratamiento de las alteraciones del desarrollo neuromuscular. Publ Inst Clim Hidrol Univ Coimbra 1987; 29: 5960 
8. San Martín I, Armijo F, San Martín J. Efectos del agua magnetizada sobre concreciones. Publ Inst Clim Hidrol Univ Coimbra 1987; 29: 73-74

9. San Martín J. Situación actual de la terapia del agua mineral en enfermedades respiratorias y otorrinolaringológicas. Acta Otorhinolaryngol Ital 1996; 16(6 Suppl 55): 68-72.

\section{Libros}

1. San Martín J. Relación entre la Conductividad Específica y la Densidad relativa de las Aguas Minero-Medicinales y su Residuo Seco [tesis]. Madrid: Ed. Servi-Off, 1975

2. Armijo M, San Martín J. Sauna. Madrid: Ed. Oteo, 1976

3. Armijo M, San Martín J. La Salud por las Aguas Termales. Madrid: Ed. Edaf, 1984

4. Armijo M, San Martín J. Hidroterapia. Madrid: SEHM, 1986-91

5. Molina A, Parreño J, San Martín J, Robles E, Moret A (Ed.). Medicina de Rehabilitación. Amsterdan: Excepta Médica, 1991

6. Molina A, Parreño J, San Martín J, Robles E, Moret A (Ed.). Rehabilitation Medicine. Amsterdan: Excepta Médica, 1991

7. Armijo M, San Martín J. Curas Balnearias y Climáticas. Talasoterapia y Helioterapia. Madrid: Ed. Complutense, 1994

\section{Capítulos de Libros}

1. San Martín J. Clasificación de las aguas mineromedicinales de Galicia. Indicaciones terapéuticas. In: El Termalismo en Galicia en la Década de los ochenta. Pontevedra: Xunta de Galicia, 1988: 171-184

2. San Martín J, Crespo PV, Maraver F. Estudio morfológico y microanalítico de la flora autótrofa C. Aguas sulfuradas. In: El Termalismo en Galicia en la Década de los ochenta. Pontevedra: Xunta de Galicia, 1988: 201-206

3. San Martín J. El termalismo en los reumatismos psicógenos. In: El Termalismo en Galicia en la Década de los ochenta. Pontevedra: Xunta de Galicia, 1988: 303-313

4. San Martín J, Maraver F. Estudio de la influencia de las aguas de Baños de Montemayor en la gonartrosis. In: El Termalismo en Galicia en la Década de los ochenta. Pontevedra: Xunta de Galicia, 1988: 315-326 
5. San Martín J, Maraver F. Rasgos demográficos del balneario de Montemayor. In: El Termalismo en Galicia en la Década de los ochenta. Pontevedra: Xunta de Galicia, 1988: 547-554

6. San Martín J, Maraver F. Valoración de una población balnearia a través de su composición según el sexo y la edad de los agüistas. In: El Termalismo en Galicia en la Década de los ochenta. Pontevedra: Xunta de Galicia, 1988: 556-561

7. San Martín J. Desarrollo y planificación de las unidades asistenciales y docentes. In: Armijo M et al. Hidrología. Madrid: Organización Médica Colegial, 1990: $17-30$

8. Armijo M, San Martín J. La formación continuada en la especialidad. In: Armijo M et al. Hidrología. Madrid: Organización Médica Colegial, 1990: 5770

9. San Martín J, Armijo M. La formación de especialistas en las Comunidades Europeas. In: Armijo M et al. Hidrología. Madrid: Organización Médica Colegial, 1990: 71-87

10. San Martín J. Hidrología Médica. In: Rehabilitación, Conceptos básicos. Valladolid: Ed. Médica Europea, 1990: 159-176

11. San Martín J. Balneoterapia. In: Rehabilitación en Geriatría. Madrid: Ed. Médicos SA, 1991: 311-328

12. San Martín J. Clasificación de las aguas mineromedicinales y ejemplos destacados de aguas gallegas e portuguesas. In: As Termas e A Clínica Geral. Porto: Câmara Municipal de Chaves, 1991: 97-102

13. San Martín J. Piscinas de tratamiento: Higiene y Control. In: Jornadas de Aguas Minerales y Mineromedicinales en España. Madrid: ITGE, 1992: 11-18

14. San Martín J. Health Resort Medicine. Integration in Universities. In: Pratzel HG (ed.). Health Resort Medicine. München: ISMH, 1995: 45-48

15. San Martín J. Experimental Study of the Action of Hipotonic Mineral Waters on the Diuresis and the Ponderal Curve. In: Pratzel HG (ed.). Health Resort Medicine. München: ISMH, 1995: 281-287

16. Meijide R, Teijeiro J, Rodríguez JL, Montoto A, San Martín J. Evaluation de la Cure Thermale de "Caldas de Lugo" (Espagne) sur le Pathologie Rhumatique. In: Pratzel HG (ed.). Sulphur in Health Resort Medicine. München: ISMH, 1995: 93-95

17. San Martín J. Hidrología médica. In: Martínez M, Pastor JM, Sendra F (ed.). Manual de Medicina Física. Madrid: Harcourt Brace Publ. Intern, 1998: 377410 
18. San Martín J. Helioterapia, talasoterapia y climatología médica. In: Martínez M, Pastor JM, Sendra F (ed.). Manual de Medicina Física. Madrid: Harcourt Brace Publ. Intern, 1998: 411-422

19. Romero M, San Martín J. Efectos para la salud de la contaminación biótica y abiótica del agua de abastecimiento urbano. In: El Agua a debate desde la Universidad. Zaragoza: Universidad de Zaragoza, 1998: 175-184

20. San Martín J, Martínez I. Investigación hidrológica en la Cátedra de Madrid. Apuntes sobre un trabajo en curso. In: Urkía JM \& Rodríguez-Sánchez JA (coords.). Los Balnearios Españoles. Salamanca: ANET, 1998: 159-168

21. San Martín J. Técnicas actuales de tratamiento balneario. Balneocinesiterapia. In: López-Geta JA, Pinuaga JJ (ed.). Panorama actual de las Aguas Minerales y Mineromedicinales en España. Madrid: ITGE, 2000: 105-114

22. San Martín J. Aplicaciones generales sin presión. Baños con aditivos. In: Técnicas hidrotermales aplicadas a Estética Integral. Madrid: Videocinco, 2000: $72-80$

23. San Martín J. Termoterapia: Sauna y Baños de vapor. In: Técnicas hidrotermales aplicadas a Estética Integral. Madrid: Videocinco, 2000: 102-112

24. San Martín J. Importancia del calcio y el magnesio y la relación calciomagnesio en las aguas de bebida. In: Agua. El arte de buen comer. Barcelona: Academia Española de Gastronomía, 2000: 205-210

25. San Martín J. Agua mineral natural de Font d'Or. In: Agua. El arte de buen comer. Barcelona: Academia Española de Gastronomía, 2000: 211-212

26. San Martín J. Conceptos generales. Terminología. Curas balnearias como agentes terapéuticos. Bases biológicas. In: Técnicas y Tecnologías en Hidrología Médica e Hidroterapia. Madrid: AETS-ISCIII, 2006: 27-32

27. San Martín J. Balneocinesiterapia. Tratamientos rehabilitadores en piscina. In: Técnicas y Tecnologías en Hidrología Médica e Hidroterapia. Madrid: AETSISCIII, 2006: 73-77 


\section{CONTRIBUCIONES A CONGRESOS}

\section{Nacionales}

1. Reunión Nacional de la Sociedad Española de ciencias Fisiológicas. Madrid 1971

- $\quad$ Comunicaciones (3):

Efecto de algunos psicofármacos sobre el consumo de oxígeno y glucosa de cerebro de rata "in vitro" Efecto del citrato de propaxolona sobre el consumo de oxígeno de algunos tejidos de rata in vitro Efectos de la meclocualona sobre glicolisis y respiración cerebral

2. IV Jornadas Nacionales de la Sociedad Española de Rehabilitación. Valencia 1973

- Comunicación:

El dolor en los hemipléjicos

3. VI Jornadas Nacionales de la Sociedad Española de Rehabilitación. Vitoria 1976

- Ponencia:

Cinesiterapia, electroterapia e hidroterapia en geriatría

4. VII Jornadas Nacionales de la Asociación Española de Rehabilitación. Badajoz 1977

- Ponencia:

Rehabilitación en vasculopatias venosas periféricas

5. VIII Jornadas Nacionales de la Sociedad Española de Rehabilitación. Madrid 1979

- Secretaria General de las Jornadas

- Comunicación:

Hidroterapia en cervicalgias psicógenas

6. IV Symposium Nacional de Rehabilitación Laboral. Zaragoza 1979

- Ponencia:

Rehabilitación del pie traumático

7. Primera Reunión Gallega de Rehabilitación. Lugo 1981

- Ponencia invitada:

Balneoterapia en Rehabilitación

8. XVIII Jornadas de la Sociedad Castellano Leonés de Rehabilitación. Valladolid 1984

- Ponencia invitada:

Hidrología Médica y Rehabilitación

9. II Xornadas Galegas de Termalismo. Carballino - Orense 1984

- Ponencia invitada:

Situación actual de la cura balnearia

10. Primeras Jornadas de Medicina Balneoterápica de Euskadi. Cestona - Guipúzcoa 1984

- Ponencia invitada

La Cura balnearia en afecciones de aparato digestivo

11. Jornades de Medicina i Balnearis als Paisos Catalans. Caldas de Montbuy - Barcelona 1984

- Ponencia invitada

Tratamiento hidroterápico y crenoterápico de la Artrosis

12. I Jornadas Andaluzas de Termalismo. Sevilla 1985

- Ponencia invitada

Rehabilitación y Termalismo 
13. Jornadas sobre Dolor y Rehabilitación. Madrid. 1986

- Ponencia:

Hidroterapia y Balneoterapia

14. I Jornadas Técnicas Turismo y Tercera Edad. Alicante 1986

- Ponencia:

El Termalismo como prevención de la patología del envejecimiento. Técnicas Termales y Balneoterapia

15. Jornadas de Turismo Termal. Balnearios de Archena y Fortuna - Murcia 1986

- Ponencia:

Interés de la cura balnearia desde el punto de vista sanitario y turístico

16. IV Jornadas Galegas de Termalismo. Illa de Toxa - Pontevedra 1988

- Ponencia invitada

El Ambiente Balneario

17. II Jornadas Nacionales de Fisioterapia. Barcelona 1988

- Ponencia invitada

Algobacterias de las Aguas Minero-Medicinales

18. III Jornadas Nacionales de Mesoterapia. Barcelona 1988

- Ponencia invitada

Hidrología Médica y Mesoterapia

19. Encuentros Monográficos sobre Problemas del Agua. Madrid 1988

- Ponencia:

La utilización terapéutica del agua como agente tópico

20. XIV Jornada Castellano-Leonesa de Rehabilitación. Palencia 1989

- Invitada por la organización

- Participación en la Mesa Redonda

Rehabilitación, Hidroterapia y Balneoterapia

21. I Jornadas sobre el futuro del Termalismo. Calatayud 1989

- Intervención y coordinación de dos Mesas Redondas Nuevas tendencias de la Balneoterapia en aparato locomotor

La Enseñanza de la Hidrología Médica en España. Presente y futuro de la Especialidad

22. Real Academia Nacional de Medicina. Madrid 1989

- Conferencia:

Acción diurética y ponderal de las aguas débilmente mineralizadas

23. Symposium de Rehabilitación "Neuropatías Periféricas". Coslada-Madrid 1989

- Ponencia invitada

Hidrocinesiterapia en Neuropatías Periféricas

24. I Congreso Nacional en Medicina y Cirugía Estética, Mesoterapia, Láser médico quirúrgico. Madrid 1990

- Miembro del Comité Científico

- Ponencia invitada

Hipótesis sobre los mecanismos de acción en Mesoterapia

25. Jornadas sobre Agua y Salud. Salamanca 1990

- Ponencia invitada

Hidrología Médica 
26. XIV Congreso Nacional de la Sociedad Española de Rehabilitación. Madrid 1990

- Presidenta Comité Científico

27. Reunión del Consejo de Turismo. Balnerio de Arnedillo 1990

- Ponencia invitada

Hidrología Médica

28. Jornadas Nacionales de Enfermería Geriátrica y Gerontológica. Zaragoza 1990

- Ponencia invitada

Beneficios de la Balneoterapia en las afecciones del aparato locomotor en el anciano

29. Sesión Científica de la Sociedad Española de Hidrología Médica. Madrid 1990

- Organizadora de la Sesión

- Ponente

Evaluación de los resultados de la cura termal

Protocolo para el seguimiento de los pacientes en cura balnearia y Protocolo específico de exploración del aparato locomotor

30. Jornada de Hidroterapia aplicada al aparato locomotor.Sabadel 1992

- Invitada por la Organización

- Conferencia Magistral:

Hidroterapia y Balneoterapia

31. Jornadas Nacionales sobre Aguas Minerales y Mineromedicinales en España. Madrid 1992

- Presidenta del Comité Organizador

- Miembro del Comité Científico

- Ponencia:

Piscinas de tratamiento. Higiene y Control

32. III Congreso de la Sociedad Madrileña de Geriatría y Gerontología. Madrid 1992

- Ponencia invitada

Balneoterapia e Hidrología

33. Congreso de la Sociedad Española de Hidrología Médica. Balneario de Cofrentes 1992

- Presidenta del Congreso y Miembro del Comité de Honor

- Miembro del Comité Organizador

- Miembro del Comité Científico

- Ponencia invitada

Espondilitis y Balneoterapia"

34. Introducció a la Balneoterapia. Caldes de Malavella 1993

- Ponencia invitada

Classificació de les aigües minero-medicinals. Indicacions principals. Mecanismes d'acció

Patología broncopulmonare di interese termale

35. Jornada en conmemoración del Centenario de D. Juan Vilanova y Piera. Real Academia de Ciencias Exactas, Físicas y Naturales. Madrid 1993

- Ponencia invitada

La Hidrología Médica en España en la Contribución Académica al Conocimiento y Aprovechamiento de las Aguas Subterráneas en España.

36. II Jornadas Andaluzas de Termalismo. Balneario de Lanjarón 1993

- Ponencia invitada

Estudios: y aplicación de las piscinas de aguas hidrotermales 
37. Jornadas sobre Balneoterapia en Geriatría. Balneario de Fitero 1993

- Ponencia invitada

Mecanismos de acción de la Cura termal

38. Congreso Anual de la Sociedad Española de Hidrología Médica y XVI Jornadas Nacionales de la Sociedad Española de Rehabilitación. La Toja 1993

- Co-Presidenta del Comité Organizador

- Ponente y Moderadora de la Primera Ponencia Conjunta: Balneoterapia y Rehabilitación

39. Congreso Galego de Educación Familia e Desenvolvemento. Santiago de Compostela 1994

- Ponencia invitada

Balnearios Galegos e Turismo Rural

40. Jornadas Nacionales de la Sociedad Española de Hidrología Médica. Madrid 1994

- Presidenta de las Jornadas

- Miembro del Comité Científico

- Miembro del Comité de Honor

- Comunicaciones:

Crenoterapia en úlceras varicosas.

Diseño de una estrategia de comunicación para llevar al público las ventajas del tratamiento termal. En memoria de un Hidrólogo.

41. IV Reunión da Sociedade Galega de Xerontoloxia e Xeriatría. Termalismo y envejecimiento. Carballino - Arnoia 1996

- Ponencia invitada

El termalismo en la tercera Edad

42. I Reunión de Estudios Históricos sobre Balnearios, Agua y Terapia. Cestona 1996

- Ponencia invitada

Investigación Hidrológica en la Cátedra de Madrid. Apuntes sobre un trabajo en curso.

43. II Jornadas Balneoterápicas de Euskadi. Zestoa 1996

- Ponencia invitada

La terapeutica termal: su realidad, su proyección

44. Primeras Jornadas en termalismo infantil. Balneario de Liérganes 1997

- Ponencia invitada

Hidrología: Visión global. Conceptos básicos.

45. 1er Congreso Nacional Medicinas no convencionales. Madrid 1997

- Ponencia invitada

Hidrología Médica.

46. Jornadas Técnicas. $1^{a}$ Feria de Termalismo y Talasoterapia. Orense 1997

- Ponencia invitada

Cura Termal y ahorro en el gasto farmacéutico

47. Real Academia de Farmacia. Madrid 1997

- Ingreso como Académico Correspondiente

- Conferencia:

Aguas mineromedicinales y minerales naturales como agente medicamentoso.

48. XI Congreso Nacional de Fisioterapia. Toledo 1997

- Ponencia invitada

Conceptos generales de termalismo. 
49. $\quad \mathbf{1}^{\text {as }}$ Jornadas de Nutrición Práctica. Madrid 1997

- Ponencia invitada El beneficio para la salud de las Aguas Minero Medicinales

50. Congreso Ibérico sobre planificación y Gestión de Aguas. Zaragoza 1998

- Miembro del Comité Científico.

- Ponencia invitada El agua a debate desde la Universidad

51. Congreso de la Sociedad Española de Hidrología Médica. Alhama de Aragón 1998

- Miembro del Comité de Honor

- Miembro del Comité Científico

- Presidente de Mesa Redonda

- Comunicaciones:

Publicaciones periódicas en el Balneario de Mondariz

Presencia de elementos mineralizantes especiales en las aguas mineromedicinales de la Comunidad Autónoma de Madrid

Distribución geográfica de las aguas mineromedicinales de Comunidad Autónoma de Madrid

52. II Reunión de Estudios Históricos sobre Balnearios, Agua y Terapéutica. Salamanca 1999

- Ponente invitada:

Otras perspectivas para la historia balnearia: Hidrología Médica

53. XVII Jornadas de la Sociedad Gallega de Rehabilitación y Medicina Física. Vigo 1999

- Ponente invitada:

Técnicas hidroterápicas

54. Mesa Redonda sobre el balneario de Carratraca. Real Academia de Farmacia. Madrid 1999

- Ponente:

Acciones terapéuticas de las aguas del Balneario de Carratraca

55. II Jornadas Aguas Minerales y Minero-Medicinales. La Toja 1999

- Presidenta del Comité Científico

- Ponente invitada:

Técnicas actuales de tratamiento balneario. Balneocinesiterapia

56. Simposio sobre patologías del músculo y sus tratamientos. Madrid 2000

- Ponente invitada:

Tratamiento de la lesión muscular en el medio hidrotermal

57. Tratamiento rehabilitador de la osteoporosis. Madrid 2000

- Ponente invitada:

Aguas mineromedicinales envasadas en la osteoporosis

58. Jornadas Universitarias de Hidrología Médica de la A.P.U.R.F. Málaga 2000

- Co-Organizadora

59. Congreso Nacional de la Sociedad Española de Hidrología Médica. Murcia 2000

- Comunicaciones (8):

Revisión de los conocimientos actuales sobre Oligoelementos

Oligoelementos Esenciales: Biodisponibilidad y papel fisiológico

Hierro- Oligoelemento Esencial

Cobre - Oligoelemento Esencial

Selenio - Oligoelemento Esencial

Manganeso- Oligoelemento Esencial

Zinc-Oligoelemento Esencial

Resumen sinóptico de los Oligoelementos Esenciales en humanos. Legislación y recomendaciones dietéticas 
60. Mesa Redonda sobre el balneario de "El Paraiso" de Manzanera (Teruel). Real Academia Farmacia. Madrid 2000

- Ponencia:

Acciones terapéuticas de las aguas de "El Paraiso" de Manzanera (Teruel)

61. Mesa Redonda sobre el balneario de Alhama de Granada. Real Academia Nacional de Farmacia. Madrid 2001

- Ponencia:

Acciones terapéuticas de las aguas del Balneario de Alhama de Granada.

62. Jornada sobre "Las aguas mineromedicinales del manantial de los Baños". Ariño - Teruel 2002

- Ponencia invitada

Termalismo y turismo de salud. Situación actual y perspectivas de desarrollo

63. Congreso Nacional de la Sociedad Española de Rehabilitación y Medicina Física. San Sebastián 2002

- Ponencia:

Talasoterapia: algo más que ocio y relax

64. Congreso Nacional de la Sociedad Española de Hidrología Médica. Balneario de Puente Viesgo 2003

- Miembro del Comité de Honor

- Presidenta de Sesiones:

- Comunicación: Actividad cardiovascular de las aguas minerales de Firgas (Islas Canarias)

65. II Congreso de la Sociedad Española de Medicina Antienvejecimiento y Longevidad. Madrid 2003

- Ponencia invitada

Hidrología Médica y antienvejecimiento

66. Sesión Científica en la Reunión Anual de la Sociedad Española de Hidrología Médica. Madrid 2003

- Conferencia:

El Olimpo y el Mar

67. Primeras Jornadas Informativas sobre Orientación Profesional para alumnos de últimos cursos de Medicina y médicos sin especialidad. Madrid 2003

- Mesa Redonda:

Especialidades Extra-hospitalarias

68. Mesa Redonda de Aguas Mineromedicinales sobre los Balnearios de Jaraba (Zaragoza). Real Academia Nacional de Farmacia. Madrid 2003

- Ponente:

Acción terapéutica de las aguas en los Balnearios de Jaraba

69. II Jornada Nacional de la Sociedad Española de Hidrología Médica. Caldes de Malavella 2003

- Ponencia invitada

Redescubrir los efectos beneficiosos del agua

70. Mesa Redonda de Aguas Mineromedicinales sobre el Balneario de Cervantes (Ciudad Real). Real Academia Nacional de Farmacia. Madrid 2006

- Ponente:

Acción terapéutica de las aguas del Balneario de Cervantes

71. Mesa Redonda de Aguas Mineromedicinales sobre el Balneario de Puente Viesgo (Cantabria). Real Academia Nacional de Farmacia. Madrid 2007

- Ponente:

Acción terapéutica de las aguas del Balneario de Puente Viesgo 
72. Congreso Nacional de la Sociedad Española de Hidrología Médica. Oropesa del Mar 2007

- Comité de Honor y Comité Científico

73. Mesa Redonda de Aguas Mineromedicinales sobre el Balneario de Valdelateja (Burgos). Real Academia Nacional de Farmacia. Madrid 2009

- Ponente:

Acción terapéutica de las aguas del Balneario de Valdelateja

74. Mesa Redonda de Aguas Mineromedicinales sobre el Balneario de Akicún de las Torres (Granada). Real Academia Nacional de Farmacia. Madrid 2009

- Ponente:

Acción terapéutica de las aguas del Balneario de Alicún de las Torres

75. Mesa Redonda de Aguas Mineromedicinales sobre el Balneario de Baños de la Concepción de Villatoya (Albacete). Real Academia Nacional de Farmacia. Madrid 2011

- Ponente:

Acción terapéutica de las aguas del Balneario de Baños de la Concepción de Villatoya

\section{Internacionales}

1. $\quad \mathbf{1}^{\text {er }}$ Congrès de l'Association Européenne de Médecine Thermale. Merano - Italia 1975

- Comunicaciones:

Modification de la formule leucocytaire chez les arthrosiques par les bains de vapeur

Résultats immediats de la kinébalnéothérapie en piscine thermale et d'eau ordinaire chaude dans le traitement des coxarthrosiques

2. Congreso Internacional de Talasoterapia. Gran Canaria 1981

- Secretaria del Congreso

- Comunicaciones:

La Talasoterapia como terapéutica de apoyo en los síndromes de sobrecarga psíquica Mineralización predominante y relaciones iónicas de las aguas marinas y terrestres Baños de ozono en el tratamiento de la parálisis cerebral

3. VIéme Congrès de la Fédération Européenne de Medecine Physique et Readaptation. Gand - Bélgica 1982

- Comunicación:

Notre expèrience dans la réadaptation des hémiplégiques au Centre National de Réhabilitation

4. Congreso Internacional de Termalismo Médico ISMH. Ponta Delgada - Azores. Portugal 1982

- Comunicaciones:

La Thalassothérapie et le traitement du surmenage professionnel

L'hydrothérapie dans les troubles fonctionnelles en rhumatologie

Relations ioniques entre les eaux chlorurées terrestres et marines

5. Giornate di studio di Talassologia e Talassoterapia. Gran Canaria 1983

- Comunicaciones:

Relaciones catiónicas de las aguas cloruradas marinas o terrestres

Talasoterapia en la Tercera Edad

V Symposium Internacional de Reumatología. Madrid 1983

- Moderadora Mesa

6. II Giornate di studio di Talassologia e Talassoterapia. Gran Canaria 1984

- Comunicaciones:

La cure héliomarine et la cure hydrothermal associée dans le traitement du psoriasis

Cure mixte hydrothermale et héliomarine dans le cervicalgies psychogènes 
7. Encontro Luso-Espanhol de Hidrologia Medica. Caldas da Rainha - Portugal 1985

- Ponencia invitada:

Crenoterapia en los reumatismos

8. Colóquio Termal do Alto Tamega y Encontro Luso-Espanhol de Hidrologia Medica. Chaves, Vidago, Verín 1986

- Comunicaciones :

Acción de los aeroiones sobre gérmenes ambientales.

Ionización negativa en la recuperación del deportista.

Hidroterapia en el tratamiento de las alteraciones del desarrollo neuromuscular.

Hipótesis acerca del mecanismo de acción diurética de la balneación.

9. Congresso Internazionale di Aggiornamento in Medicina Termale. Firenze - Italia 1986

- Ponencia invitada:

Tecnología y metodología termale

10. I Coloquio Hispano-Portugués de Hidrología Médica. Verín - Chaves 1987

- Ponencia invitada:

Las curas balnearias en geriatría (Publicada)

11. Giornate Medicee dell'Estetica - Seccion Medicina Termale. Firenze - Italia 1987

- Ponencia invitada:

La Cura Termal en las afecciones cutáneas

12. $7^{\text {a }}$ Giornate Mondiale del Termalismo e Conferenza Permanente dell'O.M.Th. Verona - Italia 1987

- Comunicación:

Sulfur Water Flora: A S.E.M and X-Ray Microanalisis Study (Publicada)

13. International Conference on Balneotherapy. Aachen - Alemania 1987

- Comunicación:

Spa therapy in Spain

14. IV International Course on Pain Relief. Murcia 1988

- Ponencia invitada:

Balneotherapy: Its possibilities in low back pain

15. Congreso Latino de Hidrología Médica. Caldas de Malavella 1988

- Co-organizadora

- Ponencias:

Envejecimiento osteoarticular y Termalismo (Publicada)

Las curas hidrotermales en los reumatismos crónicos inflamatorios

- Comunicaciones:

Programa para el estudio de los análisis de aguas Minero-Medicinales y su clasificación (Publicada) Accidentes cerebro-vasculares y su tratamiento en ambiente Balneario (Publicada)

16. Simposium Internacional sobre Artritis Reumatoide. Madrid 1988

- Ponencia invitada:

Hidroterapía y Balneoterapia en la Artritis Reumatoide

17. Giornate internazionalle di Studio Aggiornamento in Medicina Termal. Terme di Saturnia - Italia 1988

- Ponencia invitada:

Rehabilitación en Ambiente

18. $\mathbf{X}^{\text {th }}$ Congress International Federation of Physical Medicine and Rehabilitation. Toronto 1988

- Ponencias:

Magnetotherapy

Metabolic effects of magnetic fields 
19. V V̀̀me Congres International de Mesotherapie. París 1989

- Ponencia invitada: Mesoterapia y Crenoterapia en la Artritis Reumatoide

20. Congreso Internacional Preolímpico de Medicina y Deporte. Madrid 1989

- Miembro del Comité Científico

- Presidenta Mesa Redonda

- Ponencia invitada:

El tratamiento hidroterápico y su aplicación en el deportista

21. Congreso Europeo de Medicina Física y de Rehabilitación y XXXI Congreso de la Sociedad Internacional de Hidrología Médica. Madrid 1989

- Miembro del Comité Organizador

- Comunicaciones:

Crenocinebalneoterapia y Peloterapia en cervicalgias crónicas

Composición de las aguas madres preparadas a partir de agua marina y agua mineromedicinal

Evolución del crecimiento bacteriano en aguas envasadas

Estudio del coeficiente de permeabilidad de las membranas

22. II Jornadas Internacionales de Geriatría y Gerontología. Andorra 1989

- Ponencia invitada:

Hidroterapia y Crenoterapia en envejecimiento ginecológico

23. Primer Congreso Iberoamericano de Mesoterapia. Madrid 1989

- Ponencia invitada:

Mesoterapia y Aparato Locomotor

24. VI International Rehabilitation Medicine Association Congress. IRMA VI. Madrid 1990

- Miembro del Comité de Organización del Congreso

- $\quad$ Presidenta del Comité Científico

25. XXVI ${ }^{\text {ème }}$ Congres International de la Technique Hydrothermale. Sotchi - URSS 1990

- Ponencia invitada:

Caractéristique biologique du micrrobiotope des eaux minérales de Montemayor. Etudes préliminaires de la maturité des boues péloides

Analyse chromatographique à ions des eaux minérales

26. Congreso "As Termas e a Clinica Geral". Chaves - Portugal 1991

- Ponencias invitadas:

Las termas en Galicia

Mecanismos de acción de las Aguas Minero-Medicinales

27. XX Congressus Internationalis Thalassotherapiae. Borkum - Alemania 1991

- Ponencias invitadas:

Thalassotherapy in Stress

Helio -Thalassotherapy and Spa Therapy in Psoriasis

28. Jornada Científica Internacional sobre "Actualización de los Criterios de Calidad de las Aguas Minerales Naturales. Palma de Mallorca 1991

- Ponencia invitada:

Contenido Fluorado y nitrogenado en aguas minerales naturales

29. XXVII Congrès International Societé Internacional de Techniques Hydrothermale. Hajdúszoboszló Hungría 1991

- Ponencia invitada:

Control de calidad de instalaciones Balnearias 
30. VI Congresso dell'Assoziazione Europea di Medicina Termale e Bioclimatologia. Terme Bormiesi, Bormio - Italia 1991

- Ponencia invitada:

La Medicina Termale oggi e domani in Europa

31. Jornadas sobre Aguas subterráneas y su Geología en Andalucía. Torremolinos 1991

- Ponencia invitada:

Las aguas Minerales y termales. Aspectos terapéuticos. Balnearios de Andalucía

32. XI Congress of World Hydrothermal Organization. Istanbul y Pamukkale 1992

- Miembro del Comité Científico

- Ponencia invitada:

Medical Hydrology

- Comunicaciones:

Cystic Fibrosis: Thermal Experiment in Fitero.

Revision of 30 psoriatic patiens subject to thermal treatment.

33. I Encuentro con el Termalismo en Almería. Balneario de Sierra Alhamilla - Almería 1992

- Ponencia invitada:

Indicaciones de las. Aguas Minero-Medicinales y técnicas

34. Convegno Internazional "La Medicina Termale alle Soglie del 93. Acqui Terme - Italia 1992

- Ponencia invitada:

La Medicina Termal en España

35. Congresso Internazionale: Terapia Medica e Termale nella Patologia delle vie aeree superiori ed inferiori. Terme di Saturnia - Iltalia 1993

- Ponencia invitada:

Patología broncopulmonare di interesse termale.

36. $\mathbf{X X}^{\text {émes }}$. Journées Mondiales du Thermalisme. Hammamet - Tunisie 1993

- Ponencia invitada:

Problèmes de l'hygiène dans un établissement thermal

- Comunicación:

Synergie entre le traitement thermal pharmacologique et réhabilitateur aux limphoedémes primaires des extrémités inférieures

37. Segunda Jornada Nacional de Termalismo. Topes de Collantes - Cuba 1993

- Ponencia invitada:

Organización de centros de termalismo

- Conversatorio:

Indicaciones, contraindicaciones y reacciones anormales de las aguas minero-medicinales.

- Taller:

Higiene y control de piscinas de tratamiento

- Panel:

Talasoterapia y climatoterapia: acciones sobre el organismo. Indicaciones y técnicas de aplicación.

38. XXXIV Convegno Internazionale dalla Fondazione Associazione italiana di Tecnica Idrotermale (A.I.T.I.) Tabiano Terme - Italia 1993

- Coordinadora del Comité Científico

- Ponencia invitada:

Le acque minerali sulfuree.

39. Congresso Internazionalle de Medicina Termale. Attualitá e Prospettive del Termalismo. Terme di Salice - Italia 1993

- Ponencia invitada:

La Medicina Termale in Spagna: ricerca e clínica. 
40. Premières Journées de Prévention et de Ergonomie sur le mal du dos et les lésions provoquées par les mouvements répétitives du travail. Barcelona 1993

- Ponencia invitada:

Balneoterapia y Talasoterapia para la salud de espalda.

41. XXIX Congreso de la Sociedad Internacional de Técnica Hidrotermal (SITH). La Habana - Cuba 1993

- Miembro del Comité Científico

- Presidencia de sesiones

- Ponencias invitadas:

Higiene de los Establecimientos Termales

Termalismo Curativo y Turismo de Salud

42. $\quad 3^{\text {nd }}$ World Congress of the Internacional Society of Medical Hydrology and Climatology (I.S.M.H.) Bad Wörishofen - Alemania 1994

- $\quad$ Miembro - Organizing Commettee - Vice President

- Miembro - Scientific Committee

- Presidencia de sesiones

- Ponencias invitadas:

Health Resort Medicine-Integration in Universities

Diuretic Action of Hypotonic Mineral Water - An experimental Study.

43. II Internacional Symposium "Sulfur in Health Resort Medicine". Bad Nenndorf - Germany 1994

- Miembro del Comité Científico

- Chairman Session

44. I Conferencia Europea de Ecologia y Medio Ambiente. Cátedra Estrabon de la Santa Luz. Sanlúcar de Barrameda 1994

- Ponencia invitada:

Aguas Minerales y Mineromedicinales. Balnearios y Medio Ambiente

45. XXX Congress of the International Society of Hydrothermal Technique. Sinaia - Rumania 1994

- Miembro del Comité Científico

- Presidencia de sesión

- Ponencias invitadas:

Le management des Stations Balnéaires

The future of Balneary tourist activity

46. International Congress of Physical Medicine; Balneology and Medical Rehabilitation. Bucharest Romania 1994

- Ponencia invitada:

Expectation in Balneophysiotherapy and Rehabilitation in elderly people

47. XI Convegno Grupo Otorinolaringoiatrico di Studi Termali (G.O.S.T.) sobre La Sindrome Rinosinusobronquiale: Terme di Tabiano - Italia 1995

- Ponencia invitada:

Proposte crenoterapiche dell'idrologia spagnola

48. XXXV Symposium Internazionale Associazione Italiana di Tecnica Idrotermale en colaboración con S.I.T.H. y Organización Mundial del Termalísmo. Ciinquale - Italia 1995

- Miembro del Comité de Honor

49. Symposium Internacional de Investigación en Hidrología Médica. Balneario de Mondariz 1995

- Miembro del Comité Organizador

- Ponencia invitada:

La investigación y docencia de la Hidrología Médica en España. 
50. II Congreso Internacional de Turismo de Salud y II Jornada de Termalismo. La Habana - Cuba 1996

- Presidencia de sesión

- Ponencia invitada:

La Talasoterapia, aspectos y tendencias actuales.

51. International Congress on Medical Climatology, Balneology, Hydrogeology, Hydrothermal Engineering. Federation of Independent Russian Trade-Unions \& Association of Sanatoriums and Health Resorts "Profcurort". Moscow - Rusia 1996

- Concesión de la MEDALLA DE ORO DEL TERMALISMO por la International Society of Hydrothermal Technique.

52. Congreso Internacional de la Sociedad Española de Hidrología Médica S.E.H.M. Balneario de Arnedi1lo 1996

- Presidenta del Congreso

- Miembro del Comité de Honor

- Miembro del Comité Científico

- Presidencia Sesión

- Ponencia invitada:

Los Balnearios. Bienestar y salud global. Mental \& Physical Fitness

- Comunicaciones:

Propuesta parcial de algunas condiciones mínimas definitorias de las Estaciones termales y Climáticas. Influencia de la hidroterapia sobre el eje hipófiso-adrenal y título de células blancas circulantes.

Modificaciones de la actividad coagulante del plasma en los pacientes sometidos a tratamiento hidroterápico.

53. Organization Mondiale du Thermalisme (O.M.Th.) - Assemblée Générale. París 1997

- Ponencia invitada:

État de santé du thermalisme dans le monde

54. Congresso Internazionale di Medicine Termale e Climatologia. San Pellegrino Terme - Italia 1997

- Ponencia invitada:

La terapia termale nelle malattie reumatiche

55. Jornadas Técnicas Transnacionales de Análisis de las necesidades formativas en el Sector Termal. Balneario Hervideros de Cofrentes 1998

- Ponente invitada

Área Termalismo

56. Congreso Ibérico sobre Planificación y Gestión de Aguas. Zaragoza 1998

- Miembro del Comité Científico

- Ponencia invitada:

Efectos para la salud de la contaminación biótica y abiótica del agua de abastecimiento urbano

57. 33rd World Congress of the Internacional Society of Medical Hydrology and Climatology (I.S.M.H.) Karlovy Vary - Czech Republic 1998

- Vice-President of the Congress

- Nombramiento de Miembro de Honor de la ISMH

- Chairwoman

- Ponencia invitada: Health Resort Medicine II

- Poster Contribution to the study of the mineral waters in the Community of Madrid"

58. $\mathbf{X X I}^{\mathrm{e}}$ Congrès International de Thalassothérapie. Hammamet - Tunez 2000

- Presidencia de sesión

- Ponencia invitada:

La thalassothérapie: de l'Espagne au Caucase 
- Comunicaciones:

Sea water therapy in Galicia (Spain)

Four years experience in thalassotherapy center "La Perla" San Sebastián (España)

Video-Film sobre: Centre de Thalassothérapie "La Perla" San Sebastián (Spain)

59. I Congreso Internacional de Rehabilitación y Termalismo. Santiago del Estero - República Argentina 2000

- Ponencias invitadas:

Hidrología Médica

Termalismo en la Universidad

60. 35th Int'1 Seminar on Hydrotherapy, thermal and mineral waters. Société Internationale de Technique Hydrothermale. Tehran - Iran 2000

- Ponencia invitada:

Mineral waters: different types and its action in the human body

61. Aqua è vida. Expovita Termal 2000. Coimbra - Portugal 2000

- Ponencias invitadas

Termalismo no tratamento das doenças reumatismais

Qualidade dos estabelecimientos termais.

62. XVII Jornada Mundial de Termalismo y I Congreso Nacional de Técnicas Hidrotermales, Pereira Colombia 2001

- Ponencias invitadas:

Curas balnearias: Agente terapéutico y turístico.

Usos y aplicaciones de lodos y peloides (Dermatología Termal- Talasoterapia)

63. 34th Word Congress of the International Society of Medical Hydrology and Climatology. Budapest Héviz.- Hungría 2002

- Presidencia sesión

- Ponencia invitada:

Physiology of aquatic therapy: Resistance factors

64. First World Congress of Thalassotherapy. "Construisons ensemble la Thalassothérapie du 3ème Millénaire. Agadir - Marruecos 2003

- Miembro of the International Advisory Board

- Ponencia invitada:

The Olympus and the Sea

65. Congreso Transpirenaico de Medicina Física y Rehabilitación y Reunión Científica Conjunta de las Sociedades Española y Francesa de Hidrología Médica. Facultad de Medicina. Toulouse - Francia 2003

- Presidencias de sesiones

66. 40 Congreso Internacional de la SITH, Cuntis - Pontevedra 2008

- Miembro of the International Advisory Board

- Ponencia invitada:

Hygienic and sanitary aspects 


\section{TESIS / TESINAS}

\section{Tesis Doctorales dirigidas}

Francisco Maraver Eyzaguirre. Consideraciones sobre las Acciones Terapéuticas de las Aguas Minero-Medicinales de Baños de Montemayor. 1986

Juan Carlos San José Rodríguez. Estudio Histórico Científico del Balneario de Fuencaliente. 1990

$\mathrm{M}^{\mathrm{a}}$ Angeles Ceballos Hernansanz. Estudio Histórico-Científico de las Aguas Minero-Medicinales de Baños de Montemayor. Cáceres. 1991

Mª Jesús del Castillo Campos. Historia del Balneario de Mondariz hasta 1936. 1992

Raquel Bejerano Álvarez. Estudio sobre las aguas de Fuensanta y su antiguo Balneario. 1996

Inés Martínez Galán. Balnearios y Manantiales de Aguas Minero-Medicinales de la Comunidad Autónoma de Madrid. 1997

Alberto López Rocha. Balneario de Fortuna. 1998

$\mathrm{M}^{\mathrm{a}}$ Luisa Gonzalo Ruiz. Estudio del Balneario de Alhama de Aragón (Zaragoza). 1998

Estrella Cuenca Giralde. Influencia de la crenoterapia con aguas bicarbonatadas sulfatadas en el estrés oxidativo de una población balnearia. 2003

Eduardo Navarro García. Contribución al estudio de los balnearios de las Islas Canarias: aguas minerales de Firgas. 2004

María Dolores González Céspedes. Repercusión de la crenoterapia en la autopercepción de salud y capacidad funcional del anciano. 2004.

$\mathrm{M}^{\mathrm{a}}$ Concepción Serrano Moreno. Estudio experimental sobre aspectos vegetativos de gérmenes en aguas mineromedicinales y siembra directa / filtración por membrana del agua del manantial e instalaciones crenoterápicas del Balneario de Alange. 2004 


\section{Tesinas}

Iluminada Corvillo Martín. Acción de los Aeroiones sobre los Gérmenes Ambientales. 1984

Manuel Andrés Perea Horno. Revisión de la pequeña Hidroterapia concebida por Sebastián Kneipp. 1985

Luis Bernardos García. Real Sitio de La Isabella y Baños de Sacedón: Origen y Evolución Histórica. 1986

Victoria Cuartero Revilla. Consideraciones sobre la Psoriasis y su Tratamiento con especial referencia al Crenoterápico. 1989

Raquel Bejerano Alvarez. Agua de Fuensanta. 1993

Raquel Perez-Bustamante Ilander. La Casa de Baños-Hospital de Caridad de las Caldas de Oviedo en los siglos XVIII y XIX. 1998 